\title{
Two Kinds of Attention in Pavlovian Conditioning: Evidence for a Hybrid Model of Learning
}

\author{
Mark Haselgrove \\ University of Nottingham \\ John M. Pearce
Cardiff University
}

\author{
Guillem R. Esber \\ Johns Hopkins University
}

Peter M. Jones

University of Nottingham

\begin{abstract}
Four appetitive Pavlovian conditioning experiments with rats examined the rate at which the discrimination between compounds $\mathrm{AY}$ and $\mathrm{AX}$ was solved relative to the discrimination between compounds $\mathrm{AY}$ and BY. In Experiments 1 and 2, these discriminations were preceded by training in which A and $\mathrm{B}$ were continuously reinforced and $\mathrm{X}$ and $\mathrm{Y}$ were partially reinforced. Consistent with the Pearce and Hall (1980) model, the results showed that the AY/AX discrimination was solved more readily than the AY/BY discrimination. In Experiments 3 and 4, the discriminations were preceded by feature-positive training in which trials with $\mathrm{AX}$ and BY signaled food but trials with $\mathrm{X}$ and $\mathrm{Y}$ did not. Consistent with the Mackintosh (1975) model, the results showed that the AY/BY discrimination was solved more readily than the AY/AX discrimination. These results are discussed with respect to a hybrid model of conditioning and attention.
\end{abstract}

Keywords: partial reinforcement, continuous reinforcement, feature positive, predictive validity, attention, associability

Central to many theories of learning is the assumption that variation in stimulus processing determines the degree to which an association between a conditioned stimulus (CS) and an unconditioned stimulus (US) will be modified. Perhaps most influentially, Rescorla and Wagner (1972) proposed that associative learning is determined by the extent to which the CS and the US are processed. According to Rescorla and Wagner, US processing is variable and is a function of the discrepancy between the magnitude of the US and the total associative strength of all stimuli present $(\lambda-\Sigma V)$. Effects such as blocking (Kamin, 1968), conditioned inhibition (Pavlov, 1927), and the influence of degrading the contingency between the CS and US (Rescorla, 1968) were all cited as phenomena that supported the notion that learning is influenced by variations in US processing. In contrast, processing of the CS $(\alpha)$ was assumed to be fixed and determined by the physical properties of the CS. Although sympathetic to the idea that CS processing, or attention, ${ }^{1}$ may vary as a consequence of

This article was published Online First August 16, 2010.

Mark Haselgrove and Peter M. Jones, School of Psychology, University of Nottingham, Nottingham, England; Guillem R. Esber, Department of Psychological and Brain Sciences, Johns Hopkins University; John M. Pearce, School of Psychology, Cardiff University, Cardiff, Wales.

Experiment 1 was supported by a grant awarded to John M. Pearce from the Biotechnology and Biological Sciences Research Council of the UK (BBSRC). Experiments 2 to 4 were supported by a BBSRC new investigator grant to Mark Haselgrove.

Correspondence concerning this article should be addressed to Mark Haselgrove, School of Psychology, University of Nottingham, University Park, Nottingham NG7 2RD, England. E-mail: Mark.Haselgrove@ Nottingham.ac.uk experience (Wagner \& Rescorla, 1972), no attempt was made to formalize this mechanism.

Since the publication of the Rescorla-Wagner (1972) model, however, there has been considerable interest in the idea that CS processing, like US processing, varies as a consequence of learning, and efforts have been made to specify the conditions under which this variation takes place. Two theories, in particular, have come to dominate the study of CS processing. The first, by Mackintosh (1975), proposed that a CS will be processed more when it is a good predictor of a US than when it is a poor predictor of a US (see also Kruschke, 2001; Lovejoy, 1968; Sutherland \& Mackintosh, 1971; Zeaman \& House, 1963). The second, by Pearce and Hall (1980; see also Pearce, Kaye, \& Hall, 1982; Schmajuk, Lam, \& Gray, 1996), proposed the opposite: that a CS will be processed more when it is followed by unpredictable events (such as the US) than when it is followed by predictable events. As we shall see, relatively good evidence supports both of these diametrically opposite theories. We begin by considering the theory proposed by Mackintosh. According to the Mackintosh model, learning proceeds according to the individual error-term equation proposed by Bush and Mosteller (1951), in which the change in the strength of the association between CS A and the US $\left(\Delta \mathrm{V}_{\mathrm{A}}\right)$ is determined by Equation 1:

$$
\Delta \mathrm{V}_{\mathrm{A}}=\alpha \cdot \theta \cdot\left(\lambda-\mathrm{V}_{\mathrm{A}}\right)
$$

Here, the error term $\left(\lambda-V_{A}\right)$ is the discrepancy between the magnitude of the US $(\lambda)$ and the current associative strength of the

\footnotetext{
${ }^{1}$ We use the terms attention and associability interchangeably to reflect the amount of processing resources that are devoted to a CS.
} 
$\mathrm{CS}\left(\mathrm{V}_{\mathrm{A}}\right) . \theta$ is a learning rate parameter, determined by the properties of the US. Most important for the Mackintosh model, $\alpha$ is a variable CS-processing parameter that increases when the CS is the best available predictor of the US and decreases when the CS is not the best available predictor of the US. The rules proposed by Mackintosh for determining these increases and decreases are shown in Equations $2 \mathrm{a}$ and $2 \mathrm{~b}$ :

$$
\begin{gathered}
\Delta \alpha_{\mathrm{A}}>0 \text { if }\left|\lambda-\mathrm{V}_{\mathrm{A}}\right|<\left|\lambda-\mathrm{V}_{\mathrm{X}}\right| \\
\Delta \alpha_{\mathrm{A}}<0 \text { if }\left|\lambda-\mathrm{V}_{\mathrm{A}}\right| \geq\left|\lambda-\mathrm{V}_{\mathrm{X}}\right|
\end{gathered}
$$

where $\mathrm{V}_{\mathrm{X}}$ is the sum of the associative strength of all available CSs, minus $\mathrm{V}_{\mathrm{A}}$. The size of the change in $\alpha$ is assumed to be proportional to the magnitude of the inequalities in Equation 2.

The Mackintosh (1975) model provides an explanation for standard conditioning effects such as blocking and overshadowing, as well as phenomena that are not anticipated by the RescorlaWagner (1972) model, such as learned irrelevance and the intradimensional-extradimensional shift effect (for reviews, see Le Pelley, 2004; Pearce, 2008, pp. 80-86; Pearce \& Mackintosh, in press). In a recent series of experiments, we have provided further evidence for the Mackintosh model (Dopson, Esber, \& Pearce, 2010; Pearce, Esber, George, \& Haselgrove, 2008). In Dopson et al.'s (2010) Experiment 1, for example, pigeons were required first to solve two true discriminations in which two compounds signaled food $(\mathrm{AX}+, \mathrm{BY}+)$ and two did not $(\mathrm{CX}-$, $\mathrm{DY}-$ ). According to the theory proposed by Mackintosh, this training will increase attention to A and B because these CSs are good predictors of the US, whereas attention to X and Y should be slight because these CSs are irrelevant to the solution of the discrimination and are therefore poor predictors. This was tested by confronting the pigeons with an $\mathrm{AY}+, \mathrm{AX}-, \mathrm{BY}-$ discrimination. If, as a consequence of the solution of the true discriminations, attention to $\mathrm{A}$ and $\mathrm{B}$ was higher than to $\mathrm{X}$ and $\mathrm{Y}$, then the solution of the subdiscrimination that involves A and B (i.e., AY+ vs. BY-) should proceed more rapidly than the subdiscrimination that involves $\mathrm{X}$ and $\mathrm{Y}$ (i.e., $\mathrm{AY}+$ vs. $\mathrm{AX}-$ ). This was precisely the result observed by Dopson et al. A particularly noteworthy feature of the test discrimination used by Dopson et al. is that it permits an evaluation of the attention paid to stimuli that have very different associative strengths. By the end of the initial training, the associative strengths of $\mathrm{A}$ and $\mathrm{B}$ will be far higher than those of $\mathrm{X}$ and $\mathrm{Y}$. However, presenting these stimuli together as compounds $\mathrm{AX}$ and BY ensures that their total associative strengths will be at the same high level. Standard models of conditioning (e.g., Pearce, 1987; Rescorla \& Wagner, 1972) therefore predict that these compounds will both lose the same amount of associative strength on each nonreinforced trial. During the solution of the AY,$+ \mathrm{AX}-, \mathrm{BY}-$ discrimination, therefore, any difference in the rate at which the $\mathrm{AY}+, \mathrm{AX}-$ discrimination is solved relative to the $\mathrm{AY}+, \mathrm{BY}-$ discrimination can be assumed to result from differences in the attention that is paid to these stimuli.

Despite the success of the Mackintosh (1975) model, Dickinson (1980) has noted "that there is something intuitively implausible about the central idea of Mackintosh's theory" (p. 153). Dickinson argued that organisms would be far better suited to devoting their processing resources to CSs that have an uncertain rather than certain predictive relationship with the US. Pearce and Hall (1980) agreed and argued, contrary to the Mackintosh model, that CSs will be better processed when they are an inaccurate rather than an accurate predictor of the events that follow them. According to Pearce and Hall, the change in the strength of the association between CS A and the US $\left(\Delta \mathrm{V}_{\mathrm{A}}\right)$ is determined by Equation 3 .

$$
\Delta \mathrm{V}_{\mathrm{A}}=S \cdot \alpha_{\mathrm{A}} \cdot \lambda
$$

In this equation, $S$ is a fixed learning rate parameter, determined by the properties of the $\mathrm{CS} ; \lambda$ is the asymptote of conditioning, determined by the properties of the US; and $\alpha_{\mathrm{A}}$ is the associability of CS A whose value on the next trial $(n+1)$ is given by Equation 4 . The term $\left|\lambda^{n}-\Sigma V^{n}\right|$ is the absolute difference between the asymptote of conditioning and the sum of the associative strengths of all stimuli present on trial $n$.

$$
\alpha^{n+1}=\left|\lambda^{n}-\Sigma \mathrm{V}^{n}\right|
$$

One prediction that follows from Equation 4 is that a CS that is always paired with a US will lose associability because the value of $\alpha$ will eventually approach zero. There are now a number of studies that are consistent with this prediction. In Experiment 2 described by Hall and Pearce (1979), for example, continuous reinforcement of a tone with a weak US attenuated the subsequent acquisition of conditioned responding to the same tone when it signaled a stronger US (see also Ayres, Moore, \& Vigorito, 1985; Savastano, Yin, Barnet, \& Miller, 1998; Swartzentruber \& Bouton, 1986). A second prediction that follows from Equation 4 is that when a CS is intermittently paired with a US, then its associability will remain high because no matter how many trials are given, the value of $\left|\lambda^{n}-\Sigma \mathrm{V}^{n}\right|$ will always be positive. Support for this prediction can largely be found in studies using serial conditioning (Kaye \& Pearce, 1984; Pearce, Wilson, \& Kaye, 1988; Swan \& Pearce, 1988; Wilson, Boumphrey, \& Pearce, 1992). For instance, Kaye and Pearce (1984) presented a continuously reinforced group of rats with the sequence light-tone-food, and a partially reinforced group received training with the same sequence intermixed among nonreinforced trials with the light by itself. Evidence that the latter schedule maintained the associability of the light was revealed in a test phase when the light was paired directly with food and conditioning progressed more rapidly for the partially reinforced than the continuously reinforced group.

It should be apparent from the preceding discussion that there exists - at both a theoretical and an empirical level-a contradiction. On the one hand, we have Mackintosh's (1975) theory, which stipulates that CSs that are good predictors of the US will come to be better processed than stimuli that are poorer predictors of the US, and a number of studies have supported this stipulation. On the other hand, we have the theory proposed by Pearce and Hall (1980) that, to the contrary, stipulates that stimuli that are poor predictors of the US will come to be better processed than stimuli that are good predictors of the US, and again, a number of studies have supported this stipulation. To resolve this contradiction, it has been suggested that the associability of a stimulus is affected by two processes, and it is the net outcome of the interaction between these processes in any conditioning task that determines whether the associability of a stimulus is high or low (Le Pelley, 2004, in press; Pearce, George, \& Redhead, 1998; Pearce \& Mackintosh, in press). A common assumption of these theories, which differ in detail, is that on every conditioning trial, a calculation is made about how well each CS predicts the US (Mackintosh, 1975) and 
about the extent to which each CS is followed by an accurately predicted US (Pearce \& Hall, 1980). According to Le Pelley's (2004) theory, for example, the product of these terms is then used to determine the associability of each CS on the subsequent trial. We return to a detailed discussion concerning this class of theory in the General Discussion section; for the present, we focus on whether it is justifiable to assume that the changes in associability advocated by Mackintosh and by Pearce and Hall take place simultaneously in any conditioning paradigm. An alternative possibility is that these changes do not occur at the same time but are influenced by the species being tested and by the manner in which it is being tested. For example, some of the most convincing evidence that uniquely supports the principles advocated by Mackintosh comes from studies with pigeons, whereas there is relatively little evidence with this species that associability is influenced by the principles put forward by Pearce and Hall. Alternatively, demonstrations that stimuli that are good predictors of reinforcement maintain a low associability have normally involved a final test in which the stimulus by itself is paired with a US. In contrast, demonstrations that such stimuli are high in associability involve test discriminations between compounds composed of two elements. The possibility must be considered, therefore, that the rules proposed by Mackintosh and by Pearce and Hall are not effective simultaneously but instead are effective to different degrees under different circumstances and with different species. If this is correct, then a fundamental assumption of hybrid theories of associability would be undermined. The purpose of the four reported experiments is to address this issue by examining whether it is possible to obtain evidence that the associability of stimuli is governed by the principles put forward by Pearce and Hall, as well as by Mackintosh, in circumstances that are as similar as possible.

Four appetitive conditioning experiments were conducted using rats and four auditory stimuli, A, B, X, and Y (see Table 1). For the first two experiments, the initial training was intended to modify the attention paid to the four stimuli according to the principles put forward by Pearce and Hall (1980). A and B were paired consistently with food, whereas $\mathrm{X}$ and $\mathrm{Y}$ were paired intermittently with food. This treatment was expected to result in the associability of $\mathrm{A}$ and $\mathrm{B}$ being lower than that of $\mathrm{X}$ and $\mathrm{Y}$. For the second two experiments, the rats received an $\mathrm{AX}+, \mathrm{X}-$ and a $\mathrm{BY}+, \mathrm{Y}-$ discrimination, which according to the theory of Mackintosh (1975) will result in the associability of A and B being greater than that of $\mathrm{X}$ and $\mathrm{Y}$. To compare the effectiveness of these two treatments, all the experiments concluded with a test discrimination that was intended to evaluate the associability of the four stimuli. This test was based on the one described by Pearce et al. (2008; see also Dopson et al., 2010). It is important to emphasize that in all four experiments A and B were consistently paired with food, whereas $\mathrm{X}$ and $\mathrm{Y}$ were intermittently paired with food. Moreover, in Experiments 2 and 3 steps were taken to ensure that the methods of training in Stage 1 were as similar as possible and an identical method of testing was used in Stage 2. Finally, the same species was used for all of the experiments. If changes in associability take place according to the rules of Mackintosh under one set of circumstances and according to the rules of Pearce and Hall under a different set of circumstances, then the similarity of the methods of training and testing in the four experiments should ensure that they have a similar influence on the associability of the stimuli, and consequently the outcome from Experiments 1 and 2 will be similar to that for Experiments 3 and 4 . In contrast, if changes in associability take place according to both sets of rules in any conditioning procedure, then the outcome from Experiments 1 and 2 will be different than that for Experiments 3 and 4.

\section{Experiment 1}

During Stage 1, all rats were given appetitive Pavlovian conditioning in which two stimuli were followed on each trial by a food pellet, $\mathrm{A}+$ and $\mathrm{B}+$, and two stimuli were followed on half of the trials with a food pellet, $\mathrm{X} \pm$ and $\mathrm{Y} \pm$. During Stage 2, the rats were separated into two groups for testing with a discrimination. For Group AY-BY, this discrimination was of the form AY+, BY-; for Group AY-AX, the discrimination was of the form $\mathrm{AY}+$, AX-. According to the theory proposed by Pearce and Hall (1980), during Stage 1 the associability of $\mathrm{A}$ and $\mathrm{B}$ should fall as a consequence of their continuous reinforcement, but the associability to the partially reinforced $\mathrm{X}$ and $\mathrm{Y}$ should be maintained. The $\mathrm{AY}+, \mathrm{BY}-$ discrimination that must be mastered by rats in Group

Table 1

\begin{tabular}{|c|c|c|}
\hline $\begin{array}{l}\text { Experiment and } \\
\text { group }\end{array}$ & Stage 1: Pretraining & Stage 2: Test discrimination \\
\hline \multicolumn{3}{|l|}{ Experiment 1} \\
\hline Group AY-AX & $\mathrm{A}+, \mathrm{B}+, \mathrm{X} \pm, \mathrm{Y} \pm$ & $\mathrm{AY}+, \mathrm{AX}-$ \\
\hline Group AY-BY & $\mathrm{A}+, \mathrm{B}+, \mathrm{X} \pm, \mathrm{Y} \pm$ & $\mathrm{AY}+, \mathrm{BY}-$ \\
\hline \multicolumn{3}{|l|}{ Experiment 2} \\
\hline Group $12-12$ & $12 \mathrm{AB}+, 12 \mathrm{XY} \pm$ & $\mathrm{AY}+, \mathrm{AX}-, \mathrm{BY}-$ \\
\hline Group 8-16 & $8 \mathrm{AB}+, 16 \mathrm{XY} \pm$ & $\mathrm{AY}+, \mathrm{AX}-, \mathrm{BY}-$ \\
\hline Experiment 3 & $\mathrm{AX}+, \mathrm{BY}+, \mathrm{X}-, \mathrm{Y}-$ & $\mathrm{AY}+, \mathrm{AX}-, \mathrm{BY}-$ \\
\hline Experiment 4 & $\mathrm{AX}+, \mathrm{BY}+, \mathrm{X}-, \mathrm{Y}-, \mathrm{A}+, \mathrm{B}+$ & $\begin{array}{c}\mathrm{AY}: \mathrm{R} 1+\mathrm{AX}: \mathrm{R} 1-\mathrm{BY}: \mathrm{R} 1- \\
\text { and } \\
\mathrm{AY}: \mathrm{R} 2-\mathrm{AX}: \mathrm{R} 2+\mathrm{BY}: \mathrm{R} 2+\end{array}$ \\
\hline
\end{tabular}

Note. $\mathrm{A}, \mathrm{B}, \mathrm{X}$, and $\mathrm{Y}$ are auditory stimuli. Pluses and minuses are, respectively, food and no food. R1 and R2 are instrumental responses. Numbers refer to trial frequencies. See text for further details. 
AY-BY during Stage 2 should therefore be relatively hard to solve because it is based on two stimuli that have suffered a loss of associability. In contrast, the $\mathrm{AY}+, \mathrm{AX}-$ discrimination that must be mastered by rats in Group AY-AX during Stage 2 should be much easier to solve because it is based on two stimuli that still possess a high level of associability. Depending on assumptions made about the role of contextual stimuli, the Mackintosh (1975) model, in contrast, predicts there will either be no difference in the rate at which the two groups solve their discriminations or that the $\mathrm{AY}+, \mathrm{BY}-$ discrimination will be solved more readily than the $\mathrm{AY}+, \mathrm{AX}-$ discrimination. Finally, as with the experiments reported by Pearce et al. (2008), summed error-term models of learning, such as the Rescorla-Wagner model, that do not permit the processing of the CS to vary predict that the two groups should solve their discriminations at the same rate.

\section{Method}

Subjects. Thirty-two naïve male Lister hooded rats (Rattus norvegicus) supplied by Harlan Olac (Bicester, Oxon, England) served as experimental subjects. They were housed in pairs at Cardiff University (Cardiff, Wales) in a lightproof holding room in which the lights were on for $14.5 \mathrm{hr} /$ day. At the time of their arrival, their weight range was 250-300 g. Before the start of the experiment, they were gradually reduced to $80 \%$ of their freefeeding weights. They were then maintained at these weights throughout the experiment by being fed a restricted amount after each experimental session. Experimental sessions were conducted at the same time each day, during a period when the lights were on in the rats' holding room.

Apparatus. All experimental procedures were performed in eight identically specified operant boxes $(24.5 \times 23.0 \times 21.0 \mathrm{~cm})$ supplied by Campden Instruments Ltd. (Loughborough, England), which were housed in separate light- and sound-attenuating chests. A ventilating exhaust fan in each of the chests provided a background noise of $72 \mathrm{~dB}$ (C scale). Three walls of each operant box were constructed from aluminum, and a clear acrylic door served as the fourth wall. The ceiling was translucent white acrylic, and the floor was a grid made of stainless steel rods. The front aluminum wall housed a recessed food magazine $(5.0 \times 6.0 \mathrm{~cm})$ with its base located $0.5 \mathrm{~cm}$ above the grid floor. Three $5-\Omega$ loudspeakers located on the ceiling could deliver a $5-\mathrm{Hz}$ click at an intensity of $75 \mathrm{~dB}$ (C scale), a $1.8-\mathrm{kHz}$ tone at $89 \mathrm{~dB}$, a $460-\mathrm{Hz}$ buzzer at $70 \mathrm{~dB}$, and white noise at $75 \mathrm{~dB}$. On reinforced trials, a 45-mg food pellet (traditional formula, P. J. Noyes, Lancaster, NH) was delivered into the magazine tray. The magazine was covered by a clear acrylic flap hinged at the top, which the rats had to push open to gain access to the food pellets as they were delivered. A 1.0-cm-deep rectangular frame was attached to the front wall around the opening to the magazine. Set into this frame, $5 \mathrm{~mm}$ in front of the magazine flap, were three pairs of photodiode sensors in such a position that horizontal infrared beams were located 10 , 20 , and $30 \mathrm{~mm}$ above the grid floor. Appropriate circuitry permitted a Risc PC microcomputer (Acorn Computers Ltd., Cambridge, England), programmed in Arachnid (Paul Fray Ltd., Cambridge, England), to record the responses on detecting the interruption of these beams and to control the experimental events.

Procedure. All rats initially received two 30-min sessions of magazine training, during which one food pellet was delivered to the tray at regular 1-min intervals. After the end of each of these sessions, the rats remained in the conditioning chambers for another $30 \mathrm{~min}$. In the first session only, the acrylic flaps of the food magazines were taped open. All rats then received 20 sessions of acquisition training with four auditory cues, two of which were continuously reinforced $(\mathrm{A}+$ and $\mathrm{B}+)$ and two of which were partially reinforced $(\mathrm{X} \pm$ and $\mathrm{Y} \pm$ ). For half of the rats in each group, 12 trials with a tone and a buzzer were followed by the delivery of a single food pellet, and 12 trials with a clicker and a white noise were followed on half of the trials with food and on the remaining trials with no food. These relations were reversed for the other half of the rats in each group. Presentations of these stimuli were randomly intermixed with the constraint that no more than three successive trials of the same type could occur. The duration of each stimulus was $10 \mathrm{~s}$, and the interval between the end of one compound presentation and the beginning of the next, the intertrial interval, varied around a mean of $60 \mathrm{~s}$ with a range of 30-90 s. The conditioned response was the cumulative time that the rats spent with their heads in the magazine area over the 10-s trial period.

Acquisition training was followed by a discrimination test stage that lasted 12 sessions. Group AY-BY was tested with two compounds that could be discriminated by two elements that had previously been continuously reinforced $(\mathrm{AY}+, \mathrm{BY}-)$. Conversely, Group AY-AX was tested with two compounds that could be discriminated by two elements that had previously been partially reinforced $(\mathrm{AY}+, \mathrm{AX}-)$. Half the subjects in each group received 16 reinforced presentations of the buzzer and the clicker in compound, intermixed with 16 nonreinforced presentations of the tone and the clicker. Testing was similarly conducted for the remaining rats, except that the tone and the white nose constituted the reinforced compound. All other details of the procedure were identical to the previous stage.

\section{Results and Discussion}

A Type I error rate of $p<.05$ was adopted for all of the statistical tests in this and the subsequent experiments.

Conditioning during Stage 1 proceeded smoothly. The mean percent magazine activity recorded during the final session of conditioning for Group AY-AX was 49.8 and 55.7, during A/B, and during $\mathrm{X} / \mathrm{Y}$ respectively, and 48.2 and 51.3 for Group AY/BY, again, respectively. A two-way analysis of variance (ANOVA) of individual percent magazine activities with the variables of group (AY-AX vs. AY-BY) and stimulus (A/B vs. X/Y) revealed an effect of stimulus, $F(1,30)=5.85, M S E=55.73$, but no effect of group and no interaction between these variables, $F \mathrm{~s}<1, M S E \mathrm{~s}=$ $1,026.43$ and 55.73 , respectively. The mean percentage of magazine activities during the 10-s intervals before the trials with $\mathrm{A}, \mathrm{B}$, $\mathrm{X}$, and $\mathrm{Y}$ during the final session of conditioning was 24.1 for Group AY-AX and 29.6 for Group AY-BY. The difference between these means was not significant, $t(30)=0.76$.

The top panel of Figure 1 shows the mean percentage of magazine activity during $\mathrm{AY}$ and $\mathrm{AX}$ for Group AY-AX and during AY and BY for Group AY-BY across the 12 sessions of discrimination testing. Also shown in this figure is the mean percentage of magazine activity during the 10-s intervals before these trials for the two groups. Group AY-AX solved the test discrimination more readily than did Group AY-BY. To simplify the analysis of these data, discrimination ratios were computed of the form $p /(p+q)$, 


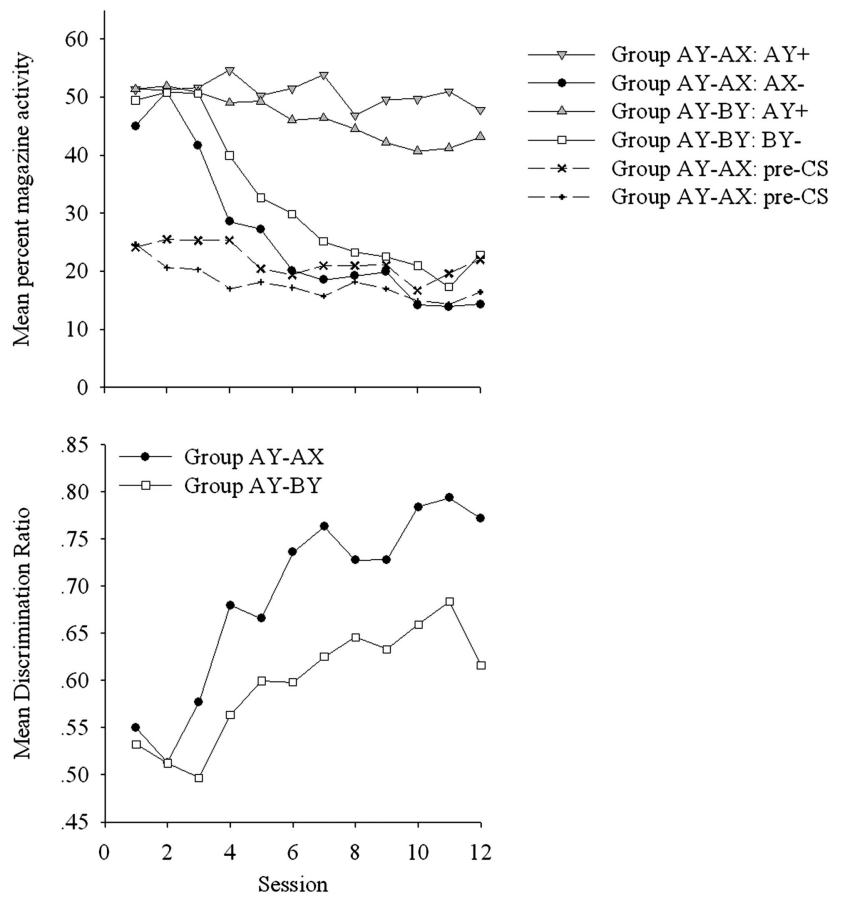

Figure 1. Results of the test discrimination from Experiment 1 for Groups AY-AX and AY-BY. The top panel shows the mean percentage of magazine activity to the test stimuli and during the pre-CS intervals across the 12 test sessions. The bottom panel shows discrimination ratios calculated from these means. $\mathrm{CS}=$ conditioned stimulus.

in which $p$ is the percentage of magazine activity during AY and $q$ is the percentage of magazine activity during AX (for Group $\mathrm{AY}-\mathrm{AX}$ ) or BY (for Group AY-BY). A discrimination ratio of 0.5 indicates no discrimination between the compounds, and a discrimination ratio of 1 indicates a perfect discrimination. These discrimination ratios for Group AY-AX and Group AY-BY are shown in the bottom panel of Figure 1. A two-way ANOVA of individual discrimination ratios with the factors of group (AY-AX vs. AY-BY) and session (1-12) was conducted. This analysis revealed an effect of session, $F(11,330)=16.00, M S E=0.01$, and of group, $F(1,30)=4.60, M S E=0.18$, but the interaction between these factors was not significant, $F(11,330)=1.57$, $M S E=0.01$

A comparison of discrimination ratios between groups is only meaningful if it is demonstrated that performance during the $\mathrm{CS}+$ is comparable in these groups. This was confirmed with a two-way ANOVA of individual percentage of magazine activities during AY with the variables of group (AY-AX vs. AY-BY) and session (1-12). This analysis revealed an effect of session, $F(11,330)=3.96$, $M S E=59.56$, but no effect of group $(F<1, M S E=4,658.78)$, and no interaction between these factors, $F(11,330)=1.77$, $M S E=59.56$. A two-way ANOVA of the mean percentage of magazine activity during 10-s intervals before the test discrimination trials was also conducted. This analysis, with the variables of group (AY-AX vs. AY-BY) and session (1-12), revealed an effect of session, $F(11,330)=2.44, M S E=86.50$, but no effect of group, $F(1,20)=2.04, M S E=2,294.01$, and no interaction between these variables $(F<1, M S E=86.50)$.
According to the theory proposed by Pearce and Hall (1980), stimuli that are good predictors of an outcome will suffer a loss of associability, whereas stimuli that are poor predictors of an outcome will have their associability maintained. It follows from these proposals that the stimuli that were initially continuously reinforced in this experiment (A and B) will have a lower associability at the outset of the test discrimination than those that were partially reinforced ( $\mathrm{X}$ and $\mathrm{Y}$ ). These predictions were confirmed because the discrimination between AY and AX was solved more readily than the discrimination between $\mathrm{AY}$ and $\mathrm{BY}$.

By the end of Stage 1, conditioned responding was more frequent during $\mathrm{X}$ and $\mathrm{Y}$ than during $\mathrm{A}$ and $\mathrm{B}$. This result is hard to explain because it is not in keeping with predictions from the majority of conditioning theories. However, a similar effect was not seen in Experiment 2, and given its unreliability we shall not comment further on this unexpected outcome of Experiment 1.

\section{Experiment 2}

The purpose of Experiment 2 was to replicate and extend the generality of Experiment 1 . Two principal changes were made to the experiment's design. First, a within-group rather than a between-groups methodology was used for the test stage of the experiment. Thus, after conditioning, rats were presented with an $\mathrm{AY}+, \mathrm{AX}-, \mathrm{BY}-$ discrimination. If more attention is paid to $\mathrm{X}$ and $\mathrm{Y}$ than to $\mathrm{A}$ and $\mathrm{B}$ at the outset of testing, then the discrimination between $\mathrm{AY}$ and $\mathrm{AX}$ will be acquired more readily than that between AY and BY. Second, Le Pelley and Beesley (2008) have noted that many of the studies that have provided evidence for the theory of learning and attention provided by Mackintosh (1975) have used training procedures with compounds of stimuli. In contrast, studies that have supported the Pearce-Hall (1980) theory have typically used training procedures in which stimuli are conditioned in isolation. Therefore, to examine whether the effects of partial and continuous reinforcement on stimulus associability are limited to stimuli that are conditioned in isolation, compounds of two stimuli were either continuously or partially reinforced (i.e., $\mathrm{AB}+, \mathrm{XY} \pm$ ) during Stage 1. After the training stage and before the discrimination test, a session in which $\mathrm{A}, \mathrm{B}, \mathrm{X}$, and $\mathrm{Y}$ were presented individually and in extinction for two trials was given to examine the strength of conditioned responding to the stimuli in isolation.

In addition, Experiment 2 included two groups of rats that differed in the detail of their partial reinforcement schedule. During the training stage of Experiment 1, the continuously reinforced stimuli ( $\mathrm{A}$ and $\mathrm{B}$ ) were presented as frequently as the partially reinforced stimuli ( $\mathrm{X}$ and $\mathrm{Y}$ ). Consequently, $\mathrm{X}$ and $\mathrm{Y}$ were paired with the US on half as many trials as A and B. It is possible that stimuli that have been paired less frequently with a US have a higher associability. If this were the case, then the subsequent test discrimination would have been easier for Group AY-AX than for Group AY-BY, which is precisely the result we observed. In this experiment, all rats were given 24 trials per session throughout Stage 1. For rats in Group 12-12, like those in Experiment 1, there were an identical number of exposures (12) to the partially (XY) and continuously $(\mathrm{AB})$ reinforced stimuli, but these stimuli were paired with the US a different number of times. On the basis of Experiment 1's results, we anticipate that the subsequent test discrimination between $\mathrm{AY}+$ and $\mathrm{AX}-$ should proceed more 
readily than the discrimination between $\mathrm{AY}+$ and $\mathrm{BY}-$. For Group 8-16, there were eight trials with $\mathrm{AB}+$ and 16 trials with $\mathrm{XY} \pm$. Rats in this group, in contrast, therefore received an identical number of pairings of the partially (XY) and continuously (AB) reinforced stimuli with the US, but these stimuli differed in the number of times they were exposed. If it is the number of times that a CS is paired with a US that determines its associability, then the test discrimination between AY and AX should proceed at the same rate as the discrimination between AY and BY. However, if differences in stimulus associability are determined by the principles advocated by Pearce and Hall (1980), then the results of the test discrimination for rats in Group 8-16 should be the same as those in Group 12-12.

\section{Method}

Subjects. The subjects were 32 experimentally naïve male rats of the same strain and from the same supplier as those in Experiment 1 . The rats were housed at the University of Nottingham (Nottingham, England) under the same conditions as the rats used in Experiment 1 with the exception that the holding room was illuminated for $12 \mathrm{hr} /$ day.

Apparatus. Eight conditioning chambers (MED Associates, St. Albans, VT; $30.0 \times 24.0 \times 20.5 \mathrm{~cm}$ high) were housed in separate light- and sound-attenuating boxes. Exhaust fans in each box provided ventilation and a background noise of $70 \mathrm{~dB}$. The two smaller walls of each chamber were constructed from aluminum, the two larger walls (one of which served as a door) and the ceiling were made of clear acrylic, and the floor was composed of a series of 19 stainless steel rods of 4.8-mm thickness, positioned $1.6 \mathrm{~cm}$ apart and parallel to the shorter walls. One of the shorter walls was equipped with a square-shaped recessed magazine, 50 $\mathrm{mm}$ wide, to which food pellets of the same type as those used in Experiment 1 could be delivered. The magazine was located 18 $\mathrm{mm}$ above the grid floor and equidistant from the two adjacent walls. An infrared beam was sent from one lateral side of the magazine and received on another. Interruption of this beam could be detected and recorded as magazine activity. The opposite wall of the chamber incorporated three loudspeakers: two squareshaped loudspeakers of $70 \mathrm{~mm}$ width located in the upper left and upper right corners of the wall and a $35-\times 70$-mm loudspeaker located $35 \mathrm{~mm}$ from the ceiling and equidistant from the two adjacent walls. The upper left loudspeaker could emit a pulsed $77-\mathrm{dB}, 8-\mathrm{kHz}$ tone, with each $0.25-\mathrm{s}$ pulse separated by a $0.25-\mathrm{s}$ silent period. The upper right loudspeaker could emit a white noise with an amplitude of $78 \mathrm{~dB}$. The rectangular loudspeaker could emit a 77-dB, 2.9-kHz tone. A relay was attached to the outside of this wall and could produce a 76-dB train of clicks such that a click occurred every $0.08 \mathrm{~s}$. Four auditory stimuli could therefore be produced (a pulsed $8-\mathrm{kHz}$ tone, a $2.9-\mathrm{kHz}$ tone, a white noise, and a clicker), each of which was $15 \mathrm{~s}$ in duration. Control of experimental events and recording of magazine activity was conducted by an attached computer programmed with the MED-PC (MED Associates, St. Albans, VT) programming language.

Procedure. Before the beginning of the experiment, auditory stimuli were assigned to serve as Stimuli A, B, X, and Y. For half the rats, the white noise and $2.9-\mathrm{kHz}$ tone were Stimuli $\mathrm{A}$ and $\mathrm{B}$; for the remaining half, the clicker and the pulsed $8-\mathrm{kHz}$ tone were Stimuli A and B. Within each of these two groups of rats, stimuli assigned to A and B were counterbalanced, as were stimuli assigned to $\mathrm{X}$ and $\mathrm{Y}$. All rats initially received a single session of magazine training, and during this 30-min session, one pellet was delivered to the magazine every $60 \mathrm{~s}$. All rats successfully consumed all pellets during this session. Rats were then trained for 16 52-min sessions. For rats in Group 12-12, there were 12 presentations per session of $\mathrm{AB}$ and 12 presentations per session of $\mathrm{XY}$. For rats in Group 8-16, each session contained eight $\mathrm{AB}+$ trials and $16 \mathrm{XY}$ trials. Two food pellets were delivered to the magazine immediately after each presentation of $\mathrm{AB}(\mathrm{AB}+)$ and half the presentations of $\mathrm{XY}(\mathrm{XY}+)$; no food was delivered after the remaining trials with $\mathrm{XY}(\mathrm{XY}-)$. These presentations were block randomized. For rats in Group 12-12, each successive block of eight trials contained four $\mathrm{AB}+$ trials, two $\mathrm{XY}+$ trials, and two $\mathrm{XY}-$ trials in a random sequence. For rats in Group 8-16, these trials were block randomized such that each successive block of six trials contained two trials of each type. The interval between the end of one auditory compound presentation and the beginning of the next, the intertrial interval, varied around a mean of $105 \mathrm{~s}$ with a range of $65-145$ s. A subsequent test session was then conducted in which rats received a block of eight training trials of the type described earlier, followed by two four-trial blocks, each containing one nonreinforced trial with each individual stimulus, followed by a final block of eight training trials. This test was designed to assess magazine responding to the four auditory stimuli in isolation. Rats were then trained for eight sessions on a final discrimination with three compounds $(\mathrm{AY}+, \mathrm{AX}-$, and $\mathrm{BY}-$ ). Each session contained $16 \mathrm{AY}+$ trials, eight $\mathrm{AX}-$ trials, and eight $\mathrm{BY}-$ trials. These were block randomized such that each successive block of eight trials contained four AY + trials and two each of $\mathrm{AX}-$ and $\mathrm{BY}-$. Intertrial intervals were the same as those used for training sessions; sessions during the final stage were $68 \mathrm{~min}$ in duration.

\section{Results and Discussion}

Conditioning in Stage 1 proceeded smoothly, and by the final session of conditioning, the mean percentage of magazine activity for rats in Group 12-12 was 57.3 during AB and 51.5 during XY. The mean percentage of magazine activity for rats in Group 8-16 was 46.1 during $\mathrm{AB}$ and 45.7 during XY. A two-way ANOVA of individual percentage of magazine activity with the variables of group (12-12 vs. 8-16) and stimulus (AB vs. XY) revealed no effect of group, $F(1,30)=2.43, M S E=475.29$; no effect of stimulus, $F(1,30)=3.05, M S E=52.33$; and no interaction between these variables, $F(1,30)=2.17, M S E=52.33$. The percentage of magazine activity during the 15 -s intervals before these trials was 12.8 and 14.4 for Groups $8-16$ and $12-12$, respectively. The difference between these means was not significant, $t(30)=0.33$.

The mean percentage of magazine activity during $\mathrm{A} / \mathrm{B}$ and $\mathrm{X} / \mathrm{Y}$ during the single test session in which $\mathrm{A}, \mathrm{B}, \mathrm{X}$, and $\mathrm{Y}$ were presented in isolation for Group 12-12 were, respectively, 46.5 and 49.2. The same measures for Group $8-16$ were 37.4 and 38.3, also respectively. A two-way ANOVA of individual percentage of magazine activity with the variables of group (12-12 vs. 8-16) and stimulus (AB vs. XY) revealed an effect of group, $F(1,30)=4.70$, $M S E=340.07$, but no effect of stimulus and no interaction between these factors $(F \mathrm{~s}<1, M S E \mathrm{~s}=97.83)$. The percentage of 
magazine activity during the 15 -s intervals before these trials was 10.8 and 14.4 for Groups $8-16$ and $12-12$, respectively. The difference between these means was not significant, $t(30)=0.99$.

The results from the test discrimination for Group 12-12 and Group 8-16 are shown, respectively, in the top left and top center panels of Figure 2. For both groups, the discrimination between $\mathrm{AY}$ and $\mathrm{AX}$ was solved more readily than the discrimination between AY and BY. To once again simplify the analysis of these data, the mean percentages of magazine activities were converted to discrimination ratios. These conversions can be seen in the lower left and lower center panels of Figure 2. A three-way ANOVA of individual discrimination ratios with the variables of group (12-12 vs. 8-16), subdiscrimination (AY/AX vs. AY/BY), and session $(1-8)$ was conducted. This analysis revealed a significant effect of session, $F(7,210)=71.88, M S E=0.01$; an effect of subdiscrimination that approached significance, $F(1$, $30)=3.58, p=.068, M S E=0.04$; and, crucially, a significant Session $\times$ Subdiscrimination interaction, $F(7,210)=4.96$, $M S E=0.01$ (all remaining $F \mathrm{~s}<0, M S E \mathrm{~s}<0.07$ ). The absence of any effect or interaction involving the variable of group necessitates that simple effects analysis be confined to the Session $\times$ Subdiscrimination interaction (the lower right-hand panel of Figure 2 shows the results of the test discrimination averaged across the two groups). This analysis revealed a difference between the subdiscriminations on Sessions 6, 7, and $8, F \mathrm{~s}(1,240)>5.71$,
$M S E \mathrm{~s}=0.01$. To demonstrate that responding during the reinforced trials with AY did not differ between the groups, a two-way ANOVA of individual percentage of magazine activity with the variables of group (12-12 vs. 8-16) and session (1 to 8) was conducted. This revealed an effect of session, $F(7,210)=2.34$, $M S E=36.86$, but no effect of group, $F(1,30)=1.74, M S E=$ $2,442.48$, and no interaction between these variables, $F(7,210)=$ 1.61, $M S E=36.86$. An identical ANOVA conducted on the individual percentage of magazine activity collected from the 15-s intervals before the test trials revealed no effect of session, $F(7$, $210)=1.03, M S E=30.31$; no effect of group, $F<1, M S E=$ 1,250.26; and no interaction between these variables $(F<1$, $M S E=30.31)$.

During Stage 1 of the experiment, rats received continuous reinforcement of an $\mathrm{AB}$ compound and partial reinforcement of an $\mathrm{XY}$ compound. One group received the same number of exposures to $\mathrm{AB}$ and $\mathrm{XY}$ (Group 12-12), whereas another group received the same number of pairings of $A B$ and $X Y$ with the food (Group $8-16)$. All rats were then required to solve an $\mathrm{AY}+, \mathrm{AX}-, \mathrm{BY}-$ discrimination. For Group 12-12, the discrimination between $\mathrm{AY}+$ and $\mathrm{AX}-$ proceeded more readily than the discrimination between $\mathrm{AY}+$ and $\mathrm{BY}-$. This finding replicates the results reported in Experiment 1 and therefore lends further support to the theory of conditioning provided by Pearce and Hall (1980). For Group 8-16, the discrimination between $\mathrm{AY}+$ and $\mathrm{AX}-$ also
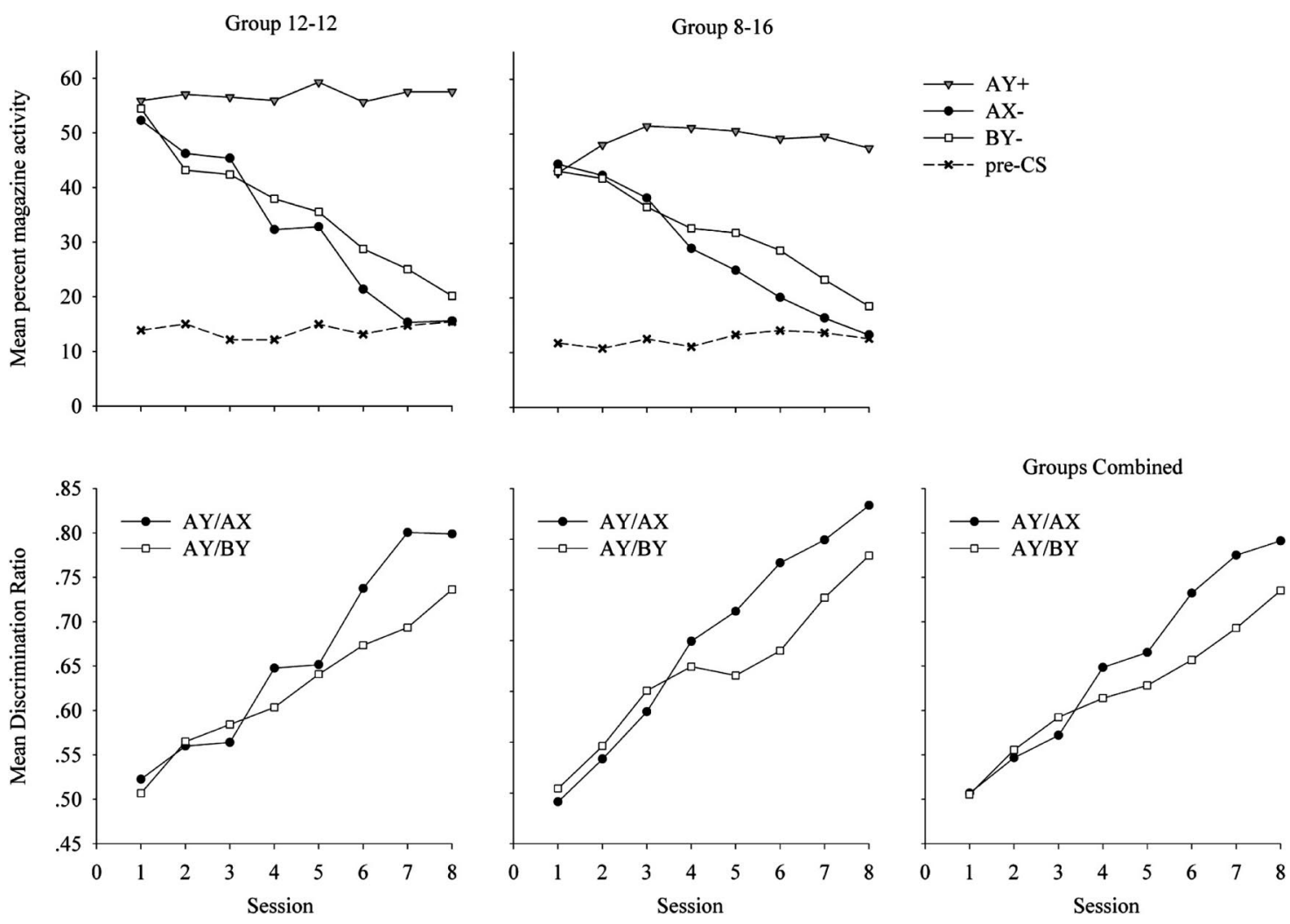

Figure 2. Results of the test discrimination from Experiment 2 for Groups 12-12 and 8-16. The top panels show the mean percentage of magazine activity to the compounds AY, AX and BY and during the pre-CS intervals across the eight test sessions. The bottom panels show discrimination ratios calculated from these means. CS $=$ conditioned stimulus. 
proceeded more readily than the discrimination between $\mathrm{AY}+$ and BY - . This result lends no support to the idea that stimuli that have been paired less often with a US differ in their associability but is again entirely consistent with the Pearce-Hall theory. In addition, this experiment lends no support to the possibility, highlighted by Le Pelley and Beesley (2008), that stimuli that are trained in compound are more likely to produce results more consistent with the theory proposed by Mackintosh (1975) than with the theory proposed by Pearce and Hall.

Pearce et al. (2008) used an AY +, AX-, BY - testing procedure with pigeons to assess differences in the associability of stimuli after the solution of a true discrimination. Their data (see also Dopson et al., 2010) provided evidence for the theory described by Mackintosh (1975). The results of Experiment 2 show, for the first time, that a similar testing procedure can be used with rats and also that it can be used to test the predictions derived from a diametrically opposite theory of learning and attention: the Pearce-Hall (1980) model. The results of Experiments 1 and 2 are further worth highlighting because they demonstrate that partially reinforced stimuli possess higher associability than continuously reinforced stimuli using a within-subjects methodology. This is both empirically and theoretically important. Honey, Good, and Manser (1998) have pointed out that if different groups receive a CS that is paired either consistently or intermittently with a US, then not only will the predictive validity of the CS differ for the two groups, but this stimulus will also occur in rather different contexts. For one group, the CS will be preceded by continuously reinforced trials, and for the other, it will be preceded by partially reinforced trials. It is conceivable that these different contexts will result in the associability of the partially reinforced stimulus being greater than the one that is continuously reinforced. Another explanation for the different effects of the two reinforcement schedules on CS associability can be developed by appeal to Hall's (2003) proposal that a US is a more effective reinforcer after it has been paired with a CS according to a partial than a continuous reinforcement schedule. If this is correct, then the results described by, for example, Kaye and Pearce (1984) can be explained by referring to a change in the effectiveness of the US rather than the CS

It is more difficult, however, to account for the results of, for example, Experiment 2 with the analysis of the effects of continuous and partial reinforcement provided by Honey et al. (1998). During Stage $1, \mathrm{AB}+$ and $\mathrm{XY} \pm$ trials were presented in a randomly intermixed order during each session. Therefore, all four stimuli occurred in the same context, and on this basis their associabilities should be equivalent. In addition, it is difficult to explain the results of Experiment 2 with the theory proposed by Hall (2003). Stimuli A, B, X, and Y were all paired with the same US during Stage 1 and thus its effectiveness as a US during Stage 2 would be predicted to be the same for the two groups. By themselves, therefore, each of these accounts incorrectly predicts that the $\mathrm{AY}+, \mathrm{BY}$ - discrimination should have been solved at the same rate as the $\mathrm{AY}+, \mathrm{AX}$ - discrimination during the test stage.

\section{Experiment 3}

Experiments 1 and 2 examined the rate at which a test discrimination was solved between two compounds, each composed of a common and a distinctive element. Both experiments revealed that the test discrimination (Experiment 1) or a subcomponent of the test discrimination (Experiment 2) was acquired more readily if the distinctive elements had previously been paired with food according to a partial than a continuous reinforcement schedule. These results, therefore, are entirely in keeping with the predictions of the Pearce-Hall (1980) model. The purpose of Experiment 3 was to establish CSs that differed in their associabilities according to principles proposed by Mackintosh (1975), while at the same time keeping the conditions of the test discrimination the same as for Experiment 2 and also, as far as possible, the conditions of the pretraining. During Stage 1, all rats were required to solve two feature-positive discriminations in which trials with $\mathrm{AX}$ and $\mathrm{BY}$ were followed by food and trials with $\mathrm{X}$ and $\mathrm{Y}$ were not. The experiments were thus matched on the stimuli that were used as A, $\mathrm{B}, \mathrm{X}$, and $\mathrm{Y}$, as well as on the details of the number of sessions of training and the number of trials within a session and their intertrial interval. It is even the case that both $\mathrm{X}$ and $\mathrm{Y}$ were partially reinforced in this experiment and $\mathrm{A}$ and $\mathrm{B}$ were continuously reinforced. Given this pretraining, the Mackintosh model predicts that the associabilities of $\mathrm{A}$ and $\mathrm{B}$ will be higher than those of $\mathrm{X}$ and $\mathrm{Y}$. This follows because A and B are better predictors of the trial outcome on $\mathrm{AX}$ and $\mathrm{BY}$ trials than are $\mathrm{X}$ and $\mathrm{Y}$, which themselves are followed by food on only $50 \%$ of the trials. The subsequent $\mathrm{AY}+, \mathrm{AX}-, \mathrm{BY}-$ test discrimination should therefore reveal that the subdiscrimination between AY and BY proceeds more readily than the subdiscrimination between AY and AX. According to the proposals of Pearce-Hall (1980), however, the associabilities of $\mathrm{A}, \mathrm{B}, \mathrm{X}$, and $\mathrm{Y}$ will all be the same. This follows because, by the end of pretraining, the trial outcome will be fully predicted on AX, BY, X, and Y trials; Equation 4 will therefore ensure that the associabilities of these stimuli will all be equivalent and relatively low. The net effect of the Mackintosh and Pearce-Hall processes should therefore be that the associability of $\mathrm{A}$ and $\mathrm{B}$ will be greater than that of $\mathrm{X}$ and $\mathrm{Y}$.

\section{Method}

Subjects and apparatus. The subjects were 16 experimentally naïve male hooded Lister rats that were maintained in an identical fashion to the subjects in Experiment 2. The apparatus was the same as that in Experiment 2.

Procedure. The rats initially received one session of magazine training, the details of which were identical to Experiment 2. During the following 16 sessions, rats were trained to solve the following discrimination: $\mathrm{AX}+, \mathrm{BY}+, \mathrm{X}-, \mathrm{Y}-$. Each 52-min session contained six trials of each type. Two food pellets were delivered to the magazine immediately after each presentation of $\mathrm{AX}$ and BY. After these sessions, a single test session was given to examine the extent to which each individual auditory stimulus controlled behavior. During this session, as during training, 24 trials occurred. The first and last blocks of eight trials were identical to those received during training, and the middle eight trials consisted of two presentations of each of the four auditory stimuli alone, in a random sequence. None of these eight trials were followed by the delivery of food. The final stage of the experiment consisted of six sessions during which rats were trained on an $\mathrm{AY}+, \mathrm{AX}-, \mathrm{BY}-$ discrimination. During these sessions, rats received presentations of $\mathrm{AY}+, \mathrm{AX}-$, and $\mathrm{BY}-$. Details of stimulus identity and counterbalancing, trial sequencing, 
session duration, and intertrial interval were identical to Experiment 2 .

\section{Results and Discussion}

Discrimination learning in Stage 1 proceeded smoothly, and by Session 16, the mean percentage of magazine activity was 53.7 during $\mathrm{AX}$ and $\mathrm{BY}$ and 13.9 during $\mathrm{X}$ and $\mathrm{Y}$. The difference between these means was significant, $t(15)=8.29$. The mean percentage of magazine activity during the 15 -s period before the onset of these trials was 5.7. During the subsequent test of A, B, X, and $\mathrm{Y}$, the mean percentage of magazine activity was 36.5 during $\mathrm{A}$ and $\mathrm{B}$ and 11.8 during $\mathrm{X}$ and $\mathrm{Y}$. The difference between these means was also significant, $t(15)=5.03$. The mean percentage of magazine activity during the 15 -s period before the onset of these trials was 6.0.

The results from the test discrimination are shown in the top panel of Figure 3. In contrast to the results from Experiments 1 and 2, the discrimination between AY and AX was solved more slowly than the discrimination between AY and BY. For the purpose of
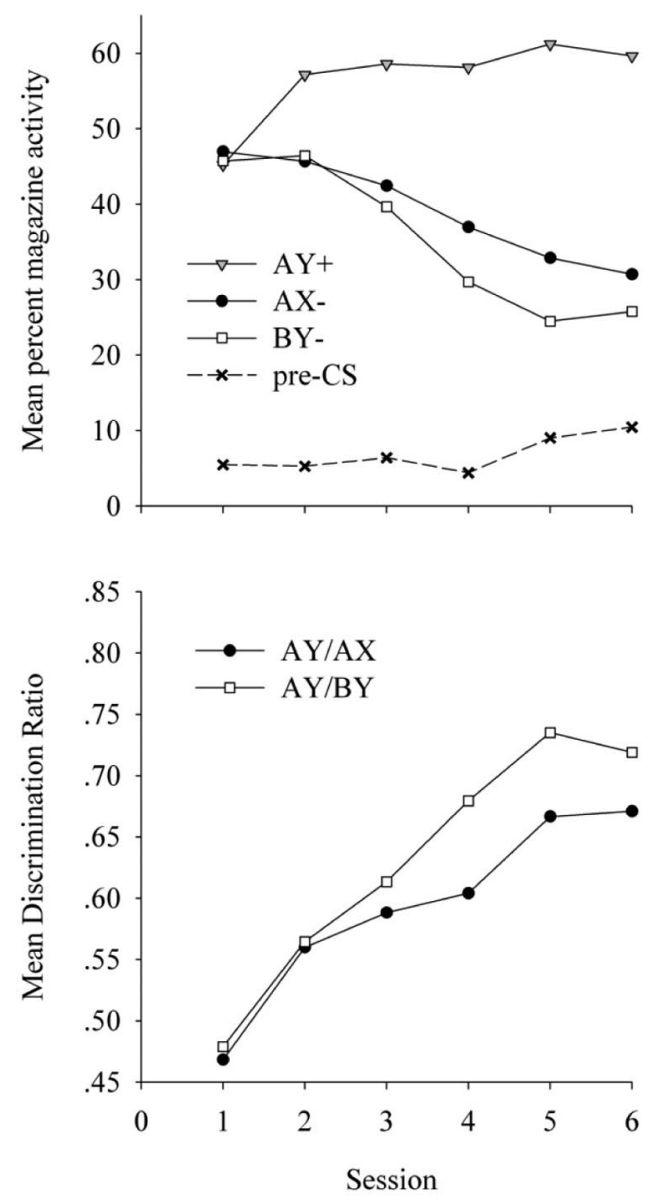

Figure 3. Results of the test discrimination from Experiment 3. The top panel shows the mean percentage of magazine activity to the compounds $\mathrm{AY}, \mathrm{AX}$ and $\mathrm{BY}$ and during the pre-CS intervals across the six test sessions. The bottom panel shows discrimination ratios calculated from these means. $\mathrm{CS}=$ conditioned stimulus. statistical analysis, the individual percentages of magazine activities were converted to discrimination ratios, and these data are shown in the lower panel of Figure 3. A two-way ANOVA of individual discrimination ratios with the variables of subdiscrimination (AY/AX vs. AY/BY) and session (1-6) revealed an effect of subdiscrimination that fell sort of significance, $F(1,15)=3.58$, $p=.078, M S E=0.02$, but a significant effect of session, $F(5$, $75)=23.26, M S E=0.01$, and, crucially, a significant Subdiscrimination $\times$ Session interaction, $F(5,75)=3.48, M S E=0.002$. Simple effects analysis revealed a difference between the discriminations on Sessions 4 and 5, Fs $(1,90)>7.42, M S E \mathrm{~s}=0.005$, and a difference on Session 6 that just failed to reach significance, $F(1$, $90)=3.66, p=.059, M S E=0.005$ (all remaining $F \mathrm{~s}<1$ ).

During pretraining, rats solved two feature-positive discriminations: $\mathrm{AX}+, \mathrm{BY}+, \mathrm{X}-, \mathrm{Y}-$. According to the theory proposed by Mackintosh (1975), as a consequence of this training, A and B should then come to command more attention than $\mathrm{X}$ and $\mathrm{Y}$. According to the theory proposed by Pearce and Hall (1980), however, by the conclusion of pretraining, there will be no uncertainty about the identity of the outcome after any of the trials. Consequently, attention to these stimuli should be equivalently low. The prediction of the Mackintosh model was confirmed in a subsequent $\mathrm{AY}+, \mathrm{AX}-, \mathrm{BY}-$ test discrimination, in which the AY, BY subdiscrimination was solved more readily than the AY, AX subdiscrimination. These results are thus consistent with the experiments described by Dopson et al. (2010). More importantly, they show the effect on attention of a process akin to that proposed by Mackintosh under circumstances comparable to Experiment 2, which itself demonstrated the effect on attention of a process akin to that proposed by Pearce and Hall. On this basis, it seems necessary to grant the assumption of hybrid models of learningthat these processes simultaneously play a role in determining the processing resources that will be directed at a CS during Pavlovian conditioning.

The results of Experiment 3 are interesting in their own right. Most analyses of the way in which feature-positive discriminations are solved have focused on the role of occasion setting and configural learning. This has been the case particularly when comparisons have been made between the solution of simultaneous and serial feature-positive discriminations (see Schmajuk, Lamoureux, \& Holland, 1998, for a review and theoretical analysis). To the best of our knowledge, there has been no analysis of the role of attention in the solution of a feature-positive discrimination. This is particularly surprising given the simplicity of the procedure and the clear predictions made by the Mackintosh (1975) model for the differing levels of attention that will, ultimately, be paid to $\mathrm{A}$ and $\mathrm{X}$. The results of Experiment 3 go some way toward addressing this omission.

\section{Experiment 4}

Although the results of Experiment 3 are consistent with the theory proposed by Mackintosh (1975), two features of the design of Experiment 3 are unsatisfactory. First, the structure of the $\mathrm{AX}+$, $\mathrm{BY}+, \mathrm{X}-, \mathrm{Y}-$ trials during pretraining means that $\mathrm{A}$ and $\mathrm{B}$ will differ from $\mathrm{X}$ and $\mathrm{Y}$ not only in terms of their predictive validity, but also in terms of (a) the number of times these CSs are exposed and (b) whether these CSs are ever presented in isolation. A slight change in the design of the pretraining for Experiment 4 over- 
comes this problem. Now, in Stage 1, all rats were given pretraining of the form $\mathrm{AX}+, \mathrm{BY}+, \mathrm{A}+, \mathrm{B}+, \mathrm{X}-, \mathrm{Y}-$. With this design, $\mathrm{A}$ and $\mathrm{B}$ continue to be the best predictors of the US on $\mathrm{AX}+$ and $\mathrm{BY}+$ trials (and, according to the Mackintosh model, should therefore be better attended to than X and Y), but now all stimuli are presented equally as frequently, as well as being presented both as part of a compound and in isolation.

Second, it is possible that the results of Experiment 3 may be accounted for not in terms of variations in CS processing, but instead, as we shall see, in terms of US processing. On the basis of experiments conducted by Rescorla $(2000,2001,2002)$, there has been a resurgence of interest in the idea that learning may be determined by US processing that is, in part, a function of the CS's own individual error term (see Equation 1) with the US rather than just a summed error term. Simulations of summed error-term models (e.g., Pearce, 1987; Rescorla \& Wagner, 1972) that we have conducted show that these theories alone predict that after training in which the associative strength of $\mathrm{A}$ and $\mathrm{B}$ reaches a higher level than $\mathrm{X}$ and $\mathrm{Y}$, the $\mathrm{AY}+, \mathrm{AX}$ - test discrimination will be solved at the same rate as the $\mathrm{AY}+, \mathrm{BY}-$ test discrimination. However, it is possible that a model that incorporates an individual error term may be able to predict the results of Experiment 3. For the $\mathrm{AY}+, \mathrm{AX}-, \mathrm{BY}-$ test discrimination to be solved, the associative strength of $\mathrm{X}$ and $\mathrm{B}$ must decline on $\mathrm{AX}-$ and $\mathrm{BY}-$ trials, respectively, to counter the excitation that is maintained or acquired by $\mathrm{A}$ and $\mathrm{Y}$ during the $\mathrm{AY}+$ trials. From the outset of the test discrimination, the associative strength of $B\left(V_{B}\right)$ will be higher than the associative strength of $\mathrm{X}\left(\mathrm{V}_{\mathrm{X}}\right)$. Consequently, the individual error term for $\mathrm{B}\left(\lambda-\mathrm{V}_{\mathrm{B}}\right)$ will be greater than the individual error term for $\mathrm{X}\left(\lambda-\mathrm{V}_{\mathrm{X}}\right)$, which should result in a greater loss of associative strength to $\mathrm{B}$ on $\mathrm{BY}$ trials than to $\mathrm{X}$ on AX trials. Simulations that we have conducted using a variety of individual error-term models (e.g., Le Pelley, 2004, pp. 198-201) confirm this intuition: After $\mathrm{AX}+, \mathrm{BY}+, \mathrm{X}-, \mathrm{Y}-$ pretraining, during the solution of an $\mathrm{AY}+, \mathrm{AX}-, \mathrm{BY}-$ test discrimination, the associative strength of the BY compound declines more rapidly than that of the AX compound, which is exactly the result we observed in Experiment 3.

A simple way to counter this argument is to point out that differences in the associative strengths of $\mathrm{X}$ and $\mathrm{B}$, from the outset of the test discrimination, cannot be the only factor that determines the rate at which the $\mathrm{AY}+, \mathrm{AX}-$ and $\mathrm{AY}+, \mathrm{BY}-$ discriminations are solved. If it were, then the results of the test discrimination from Experiment 2 (in which the associative strength of B was again, presumably, higher than that of $\mathrm{X}$ ) should have exactly matched the results of Experiment 3, which it did not. However, this counterpoint is rather unsatisfactory because it still remains questionable whether the results of Experiment 3, alone, provide evidence for the theory proposed by Mackintosh (1975). To overcome this potential problem, the test discrimination of Experiment 4 was changed. Now, instead of trials with AY, AX, and BY signaling the US or the absence of the US, these compounds signaled reinforcement after the performance of one instrumental response, but not another. During trials with AY, performance of Response 1 (R1) was reinforced, but performance of Response 2 (R2) was not. During trials with AX and BY, performance of R2 was reinforced but performance of $\mathrm{R} 1$ was not. If attention to $\mathrm{A}$ and $\mathrm{B}$ is greater than that to $\mathrm{X}$ and $\mathrm{Y}$ at the outset of the test discrimination, then the subdiscrimination between AY and BY should still be easier to solve than the subdiscrimination between $\mathrm{AY}$ and $\mathrm{AX}$ even though the discrimination is based on an instrumental rather than a Pavlovian task. However, the instrumental test discrimination cannot be solved simply by the loss of associative strength by $\mathrm{B}$ and $\mathrm{X}$. Consequently, any differences in the associative strength of $\mathrm{B}$ and $\mathrm{X}$, or indeed $\mathrm{A}$ and $\mathrm{Y}$, at the outset of the test discrimination should not influence the rate at which the subdiscrimination between $\mathrm{AY}$ and $\mathrm{AX}$ is solved relative to the subdiscrimination between AY and BY.

\section{Method}

Subjects and apparatus. The subjects were 16 experimentally naive male hooded Lister rats that were maintained in an identical fashion to the subjects in Experiment 2. The apparatus was the same as described in Experiment 2. In addition to the features previously described, each chamber was now equipped with two retractable levers (MED Associates). These levers could be either presented to the rat or fully retracted into the wall of the chamber, a state that was controlled by the experimental computer. When presented, the levers protruded $1.9 \mathrm{~cm}$ from the wall and were $4.8 \mathrm{~cm}$ wide and $0.2 \mathrm{~cm}$ thick. The levers were positioned directly beneath the two circular lamps on the wall of the chamber that contained the food magazine, $6.2 \mathrm{~cm}$ from the grid floor.

Procedure. Assignment of stimuli was carried out in the same way as for Experiment 2, and rats initially received one session of magazine training of the type previously described. During the next five sessions, rats were trained to press both levers. At the outset of each session, both levers were retracted; after $30 \mathrm{~s}$, one lever was presented for $60 \mathrm{~s}$. Thirty seconds after the retraction of this lever, the other was presented for $60 \mathrm{~s}$. This pattern continued for the duration of the 60-min session, with levers presented in a double-alternating sequence. Although either lever was available, rats were able to earn food pellets according to a schedule that was varied from session to session. For the first session, each lever press was followed by the delivery of a food pellet to the magazine. During the subsequent four lever-training sessions, lever presses were followed by food on a variable-interval schedule. The mean intervals were $3 \mathrm{~s}$ for the first session, $7 \mathrm{~s}$ for the second, and $15 \mathrm{~s}$ for the third and fourth. By the end of these sessions, the mean rate of responding was 9.2 responses per minute, and all rats pressed the levers at a rate of at least five responses per minute.

Rats were then trained for 16 52-min sessions to solve the following discrimination: $\mathrm{AX}+, \mathrm{BY}+, \mathrm{A}+, \mathrm{B}+, \mathrm{X}-, \mathrm{Y}-$. Two food pellets were delivered to the magazine immediately after each presentation of $\mathrm{AX}, \mathrm{BY}, \mathrm{A}$, and $\mathrm{B}$; no food was delivered after presentations of $\mathrm{X}$ or $\mathrm{Y}$. These presentations were block randomized such that each successive block of 12 trials contained two trials of each type, in a random sequence. Intertrial intervals were the same as for Experiment 2. Both levers were retracted during this stage of the experiment. Rats were subsequently given a single session of lever retraining that was identical to the final session of the initial lever training.

Rats were then trained for 14 sessions on a final discrimination with three compounds (AY, AX, and BY). Rats received food pellets during these compounds after "correct" lever presses, with the correct lever defined as follows: For half the rats, the left lever was correct for AY trials and the right lever was correct for AX and BY trials. For the remainder, the right lever was correct for 
AY trials and the left lever was correct for AX and BY. To receive food, therefore, rats were required to press one lever during AY trials and the alternative lever during $\mathrm{AX}$ and BY trials. Each stimulus presentation in this stage had a duration of $20 \mathrm{~s}$; both levers were presented during stimuli and were retracted at all other times. During the initial $15 \mathrm{~s}$ of each stimulus, lever presses did not result in food delivery. Two pellets were delivered to the food magazine after the first correct lever press during the final $5 \mathrm{~s}$ of each stimulus. Each 71-min session contained 16 presentations of AY, eight of AX, and eight of BY. These were block randomized such that each block of eight trials contained four with AY and two of each of the other trial types. Intertrial intervals were the same as for the previous discrimination.

\section{Results and Discussion}

Two rats failed to respond more frequently to $\mathrm{R} 1$ than to $\mathrm{R} 2$ during trials with AY in the test discrimination; these rats were therefore omitted from all analyses. Discrimination learning in Stage 1 proceeded smoothly, and by Session 16, the mean percentage of magazine activity was 60.1 during AX and BY, 57.8 during $\mathrm{A}$ and $\mathrm{B}$, and 17.7 during $\mathrm{X}$ and $\mathrm{Y}$. A one-way ANOVA of individual mean percentage of magazine activity revealed that the difference among these means was significant, $F(2,26)=52.77$, $M S E=151.02$. Bonferroni-corrected $t$ tests revealed that the difference between $\mathrm{AX} / \mathrm{BY}$ and $\mathrm{X} / \mathrm{Y}$ was significant, $t(13)=$
42.41, as was the difference between $\mathrm{A} / \mathrm{B}$ and $\mathrm{X} / \mathrm{Y}, t(13)=40.15$. The difference between AX/BY and A/B was not significant, $t(13)=2.26$. The mean percentage of magazine activity during the 15-s intervals before these trials was 10.5 .

The top left and top center panels of Figure 4 show the mean number of responses per minute on R1 and R2 during the first $15 \mathrm{~s}$ with $\mathrm{AY}, \mathrm{AX}$, and $\mathrm{BY}$ across the 14 sessions of testing. Levers were retracted in the absence of $\mathrm{AY}, \mathrm{AX}$, and $\mathrm{BY}$, hence the absence of data from the periods before these trials. The discrimination between AY and BY was solved more readily than the discrimination between $\mathrm{AY}$ and $\mathrm{AX}$ on both R1 and R2. There was an indication that the difference between the AY/AX and AY/BY discriminations was more pronounced on $\mathrm{R} 1$ than on $\mathrm{R} 2$. The lower left and center panels show discrimination ratios for the AY/AX and AY/BY subdiscriminations calculated from the mean number of responses per minute on, respectively, R1 and R2. For $\mathrm{R} 1$, these ratios took the form $\mathrm{AY} /(\mathrm{AY}+\mathrm{AX})$ and $\mathrm{AY} /(\mathrm{AY}+$ $\mathrm{BY})$. For $\mathrm{R} 2$, the ratios took the form $\mathrm{AX} /(\mathrm{AX}+\mathrm{AY})$ and $\mathrm{BY} /(\mathrm{BY}+\mathrm{AY})$. A three-way ANOVA of individual discrimination ratios with the variables of response (R1 vs. R2), subdiscrimination (AY/AX vs. AY/BY), and session (1-14) was conducted. This analysis revealed an effect of session, $F(13,169)=28.51$, $M S E=0.01$; an effect of response that just met statistical significance, $F(1,13) 4.74, p=.049, M S E=0.03$ : and, crucially, an effect of discrimination, $F(1,13)=5.85, M S E=0.06$. The Response $\times$ Discrimination interaction was not significant, $F(1$,
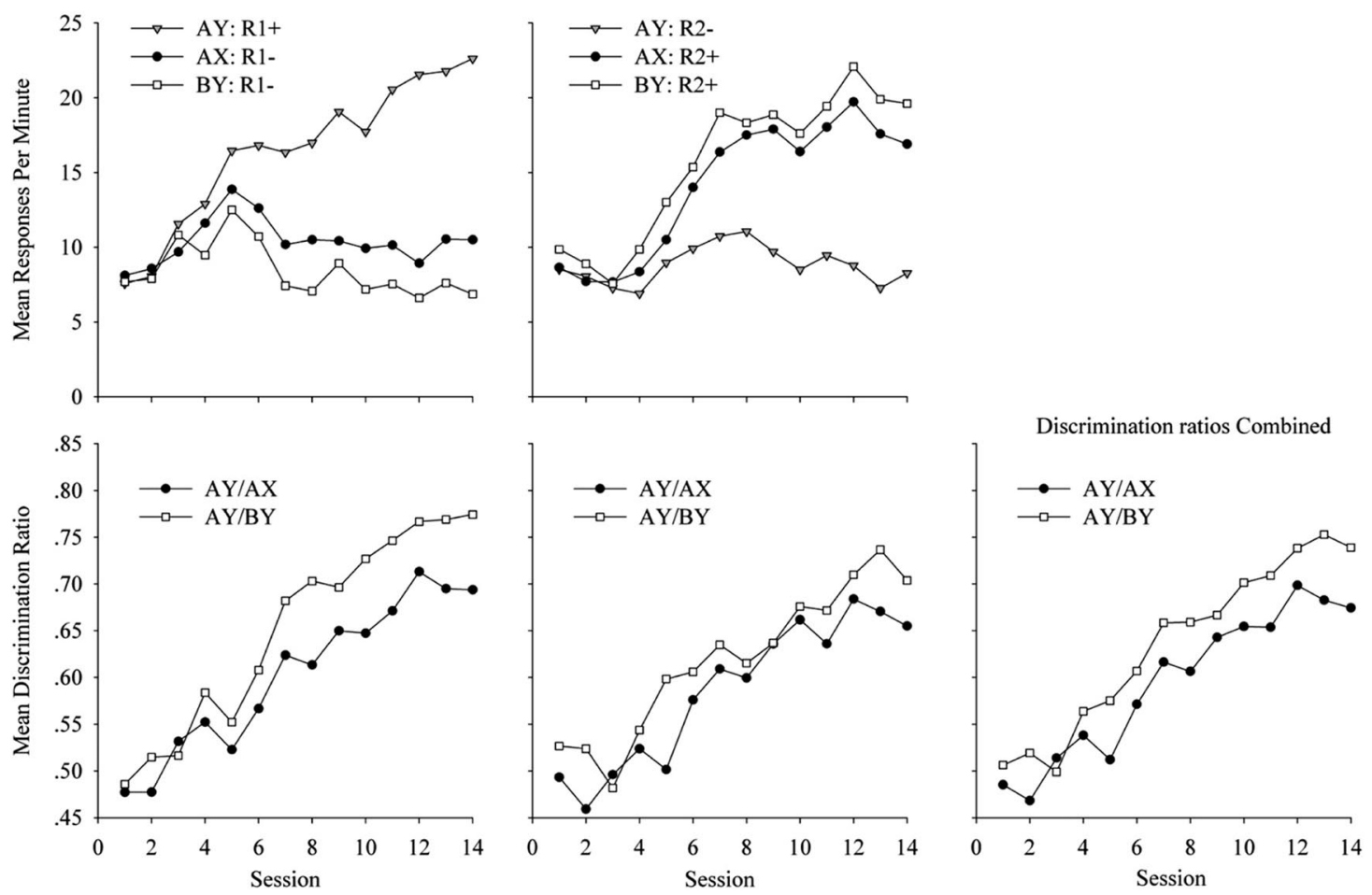

Figure 4. Results of the test discrimination from Experiment 4. The top panels show the mean rates of responding to Response 1 (R1) and Response 2 (R2) during compounds AY, AX and BY across the 14 test sessions. The bottom panels show discrimination ratios calculated from these means. 
13) $=1.96, M S E=0.01$, and neither were any of the remaining interactions, $F \mathrm{~s}(13,169)<1.04$, MSEs $<0.01$. The effect of discrimination demonstrates that, overall, the discrimination between $\mathrm{AY}$ and BY was more successful than the discrimination between $\mathrm{AY}$ and $\mathrm{AX}$, irrespective of whether performance on R1 or R2 was measured. The lower right panel shows the discrimination ratios for the AY/AX and AY/BY subdiscriminations collapsed across $\mathrm{R} 1$ and $\mathrm{R} 2$.

These results confirm and extend the results of Experiment 3. Rats were first given training in which trials with $\mathrm{AX}, \mathrm{BY}, \mathrm{A}$, and $\mathrm{B}$ were followed by food and trials with $\mathrm{X}$ and $\mathrm{Y}$ were not. Relative to $\mathrm{X}$ and $\mathrm{Y}, \mathrm{A}$ and $\mathrm{B}$ are better predictors of the trial outcome. According to the theory proposed by Mackintosh (1975), therefore, attention to A and $\mathrm{B}$ should be greater than attention to $\mathrm{X}$ and $\mathrm{Y}$. Again, the theory proposed by Pearce and Hall (1980) predicts that, by the conclusion of pretraining, there will be no uncertainty about the identity of the outcome after any of the trials. Consequently, attention to these stimuli should be equivalently low. The results of the final test discrimination confirmed the predictions of the Mackintosh modelthe discrimination between AY and BY was more successful than the discrimination between AY and AX. It is important to note that this result was observed under conditions in which the difference in the associative strength of $\mathrm{B}$ and $\mathrm{X}$ was unlikely to have contributed to the effect. During the test discrimination, AY, AX, and BY were all paired with food. Unlike in Experiment 3, therefore, B and X do not have to lose associative strength on the $\mathrm{AX}$ and $\mathrm{BY}$ trials to counter the excitation gained on AY trials. Consequently, any differences in the associative strengths of $\mathrm{B}$ and $\mathrm{X}$ from the outset of the test discrimination are unlikely to have contributed to the difference observed.

This experiment is also interesting in its own right because it demonstrates that attention acquired to a stimulus through Pavlovian conditioning can subsequently influence the extent to which this stimulus can take control of instrumental responding. This result therefore provides support for the assumption behind theories of addictive behavior that propose that via conditioning, attention increases to drug-paired cues and that this bias then subsequently influences instrumental drug-seeking behavior (e.g., Hogarth \& Duka, 2006; Robinson \& Berridge, 1993).

\section{General Discussion}

Four experiments examined the rate at which a discrimination was solved between two compounds, each composed of a common and a distinctive element. Experiments 1 and 2 revealed that the discrimination was acquired more readily if the distinctive elements had previously been paired with food according to a partial rather than a continuous reinforcement schedule. This result is consistent with the theory proposed by Pearce and Hall (1980). Experiments 3 and 4 revealed that the discrimination was acquired more readily if the distinctive elements had previously been relevant, rather than irrelevant, to the solution of a feature-positive discrimination. This result is consistent with the theory proposed by Mackintosh (1975).

The results of Experiments 1 and 2 are compatible with those of previous studies that have shown that the associability of partially reinforced stimuli is greater than that of continuously reinforced stimuli (e.g., Kaye \& Pearce, 1984). At the same time, these results have a number of advantages over most of those from previous studies. First, it has been possible to compare the effects of the two reinforcement schedules using conventional training, in which the CS is paired directly with the US, instead of less typical, serial conditioning designs. The results thus provide novel confirmation of predictions from the Pearce and Hall (1980) theory using the most direct method of training. Second, it is not possible to interpret these results by referring to explanations that can be applied to the earlier studies (e.g., Hall, 2003; Honey et al., 1998). Indeed, we are aware of only one experimental design with rats that supports the proposals of Pearce and Hall that, at the same time, is immune to these alternative interpretations (e.g., Maddux, Kerfoot, Chatterjee, \& Holland, 2007; Wilson, Boumphrey, \& Pearce, 1992).

As we noted earlier, the testing procedure used in the current experiments was based on the design of the experiments described by Pearce et al. (2008; see also Dopson et al., 2010). The experiments reported by Dopson et al. (2010), for example, showed that after the solution of a pair of true discriminations $(\mathrm{AX}+, \mathrm{BY}+$, $\mathrm{CX}-, \mathrm{DY}-$ ), pigeons solved a subsequent test discrimination between $\mathrm{AY}+$ and $\mathrm{BY}-$ more readily than a discrimination between $\mathrm{AY}+$ and $\mathrm{AX}-$. In Dopson et al.'s experiments, therefore, discrimination learning proceeded more rapidly between compounds whose distinctive stimuli had a history of being continuously followed by a US (AY and BY) than of being intermittently followed by a US (AY and AX). Experiments 3 and 4 demonstrated results consistent with this result, but as we have noted, Experiments 1 and 2 demonstrate results that are contrary to the findings of Dopson et al. To reconcile these contrary results, it seems necessary to acknowledge that CS processing is determined simultaneously by two processes, one that follows the principles suggested by Mackintosh (1975) and another that follows the principles suggested by Pearce and Hall (1980). As we have noted, a number of so-called hybrid models of CS processing have now been proposed. Pearce, George, and Redhead (1998), for example, incorporated variants of the Mackintosh and Pearce-Hall theories into a configural model of learning, and Le Pelley (2004) has incorporated variants of the same theories into an elemental model of learning. The apparent contradiction between the Mackintosh and Pearce-Hall models can be resolved if it is appreciated that the two theories act on CS processing in different ways. According to Le Pelley, the Mackintosh model allocates more attention to the CS that has the best relative predictive validity - that is to say, the CS that, relative to all other CSs, is the best predictor of the US. Le Pelley has classified this property of the CS as attentional associability. The Pearce-Hall model, however, allocates attention to the CS on the basis of its absolute predictive validity-that is to say, the extent to which it is followed by a well-predicted US irrespective of the predictive validity of other CSs. Le Pelley has classified this property of the CS as salience associability. In particular, Le Pelley (in press) reported simulations of the current Experiment 1 and Dopson et al.'s Experiment 1 with a hybrid model, and we direct the interested reader to this article for details of how, formally, a hybrid model explains, on the one hand, results consistent with the Mackintosh model and, on the other hand, results consistent with the Pearce-Hall model.

It is, however, instructive to gain an intuition for how such hybrid models of attention and learning arrive at the predictions that we have derived here. The remaining discussion will consider, more informally, how a recent hybrid model proposed by Pearce and Mackintosh (in press) can explain the present results. Pearce and Mackintosh have simplified the proposals of Le Pelley. They 
Table 2

Analysis of Experiments 1 and 3 With a Hybrid Model of Learning and Attention (Pearce \& Mackintosh, in Press)

\begin{tabular}{|c|c|c|c|c|}
\hline $\begin{array}{l}\text { Experiment/ } \\
\text { Training }\end{array}$ & $\begin{array}{c}\text { Learning rule } \\
\Delta \mathrm{V}_{\mathrm{A}}=\alpha \times \sigma \times \beta \times(\lambda-\Sigma \mathrm{V})\end{array}$ & $\begin{array}{l}\text { Attentional associability }(\alpha) \text { : } \\
\Delta \alpha_{\mathrm{A}}>0 \text { if }\left|\lambda-\mathrm{V}_{\mathrm{A}}\right|<\left|\lambda-\mathrm{V}_{\mathrm{X}}\right| \\
\Delta \alpha_{\mathrm{A}}<0 \text { if }\left|\lambda-\mathrm{V}_{\mathrm{A}}\right| \geq\left|\lambda-\mathrm{V}_{\mathrm{X}}\right|\end{array}$ & $\begin{array}{l}\text { Salience associability }(\sigma) \text { : } \\
\qquad \sigma^{n+1}=\left|\lambda^{n}-\mathrm{V}^{n}\right|\end{array}$ & $\begin{array}{l}\text { Total associability: } \\
\qquad \alpha \times \sigma\end{array}$ \\
\hline \multicolumn{5}{|l|}{ Experiment 1} \\
\hline $\mathrm{A}+\mathrm{B}+$ & $\mathrm{V}_{\mathrm{A} / \mathrm{B}}=1 \lambda$ & $\alpha_{\mathrm{A} / \mathrm{B}} \approx 1$ & $\sigma_{\mathrm{A} / \mathrm{B}} \approx .3$ & $\mathrm{~A} / \mathrm{B}=1 \times .3=.3$ \\
\hline $\mathrm{X} \pm \mathrm{Y} \pm$ & $\mathrm{V}_{\mathrm{X} / \mathrm{Y}}=.5 \lambda$ & $\alpha_{\mathrm{X} / \mathrm{Y}} \approx 1$ & $\sigma_{\mathrm{X} / \mathrm{Y}} \approx .5$ & $\mathrm{X} / \mathrm{Y}=1 \times .5=.5$ \\
\hline \multicolumn{5}{|l|}{ Experiment 3} \\
\hline $\mathrm{AX}+\mathrm{BY}+$ & $\mathrm{V}_{\mathrm{A} / \mathrm{B}}=1 \lambda$ & $\alpha_{\mathrm{A} / \mathrm{B}} \approx 1$ & $\sigma_{\mathrm{A} / \mathrm{B}} \approx .3$ & $\mathrm{~A} / \mathrm{B}=1 \times .3=.3$ \\
\hline $\mathrm{X}-\mathrm{Y}-$ & $\mathrm{V}_{\mathrm{X} / \mathrm{Y}}=0 \lambda$ & $\alpha_{X / Y} \approx .05$ & $\sigma_{\mathrm{X} / \mathrm{Y}} \approx .5$ & $\mathrm{X} / \mathrm{Y}=.05 \times .5=.025$ \\
\hline
\end{tabular}

Note. Values in bold denote total associability.

suggested that learning proceeds according to the proposals of Rescorla and Wagner (1972). Thus, on each trial the change in the strength of the association between a CS (say, A) and a US is determined by a summed error term $(\lambda-\Sigma \mathrm{V})$ that is multiplied by the product of three learning-rate parameters: $\beta$, which is fixed and is determined by the intensity of the US, and $\alpha$ and $\sigma$, which are modifiable parameters reflecting attention to the CS. The rules governing changes in $\alpha$ (attentional associability) follow those proposed by Mackintosh (1975) and are shown in Equations 2a and $2 \mathrm{~b}$. The rules governing changes in $\sigma$ (salience associability) follow a variation on those proposed by Pearce and Hall and are shown in Equation 5, where the superscripts $n$ and $n+1$ refer to the current trial and subsequent trial, respectively.

$$
\sigma_{\mathrm{A}}^{n+1}=\left|\lambda^{n}-\mathrm{V}_{\mathrm{A}}^{n}\right| .
$$

The modification to the Pearce-Hall (1980) model is interesting because now the (salience) associability of the CS is determined by its individual prediction error rather than a summed prediction error. Thus, the salience associability of the CS is for the first time determined by absolute predictive validity. ${ }^{2}$ Le Pelley (in press) has suggested that $\alpha$ can vary between the values of .05 and 1 and that $\sigma$ can vary between the values of .3 and 1 . These ranges of values allow hybrid models to be consistent with the intuition for how the Mackintosh (1975) and Pearce-Hall models distinguish themselves: The Mackintosh (attentional associability) component allows stimuli to be either fully attended to or almost fully ignored on the basis of their relevance relative to other stimuli. The Pearce-Hall (salience associability) component determines how much is to be learned about stimuli on the basis of their individual, absolute predictive validity. Finally, the total associability of a CS is given by the product of $\alpha$ and $\sigma$.

By adopting the proposals of Pearce and Mackintosh (in press), the results of Experiments 1 and 2 can be reconciled with those of Experiments 3 and 4. During Experiment 1, for example, it follows from Equation 5 that $\sigma$ for $\mathrm{A}$ and $\mathrm{B}$ after continuous reinforcement will be close to the minimum value of .3. However, because A and $\mathrm{B}$ are the best predictors of the US, Equation $2 \mathrm{a}$ will ensure that $\alpha$ will be close to the maximum value, 1 . Thus, the total associability $(\alpha . \sigma)$ for $\mathrm{A}$ and $\mathrm{B}$ will be close to .3. However, the overall associability of $\mathrm{X}$ and $\mathrm{Y}$ will be greater than this value. This prediction follows from Equation $2 \mathrm{a}$ because $\mathrm{X}$ and $\mathrm{Y}$ are also the best predictors of the US, ensuring that the value of $\alpha$ remains relatively high. At the same time, Equation 5 will ensure that $\sigma$ is close to .5 for $\mathrm{X}$ and $\mathrm{Y}{ }^{3}$ The product of $\alpha$ and $\sigma$ for $\mathrm{X}$ and $\mathrm{Y}$ will be greater than the product of $\alpha$ and $\sigma$ for $\mathrm{A}$ and $\mathrm{B}$, and the discrimination between $\mathrm{AY}$ and $\mathrm{AX}$ will then proceed more rapidly than the discrimination between AY and BY. A summary of these principles is shown in the center rows of Table 2 .

During the solution of the feature-positive discriminations in Experiment 3, for example ( $\mathrm{AX}+, \mathrm{BY}+, \mathrm{X}-, \mathrm{Y}-), \mathrm{X}$ and $\mathrm{Y}$ were intermittently followed by the US. According to Equation 5, $\sigma$ for $\mathrm{X}$ and $\mathrm{Y}$ will therefore be relatively high. However, because $\mathrm{X}$ and $\mathrm{Y}$ were not the best predictors of the US, Equation $2 \mathrm{~b}$ predicts that $\alpha$ for these stimuli will be close to zero and will result in the product of $\alpha$ and $\sigma$ for $\mathrm{X}$ and $\mathrm{Y}$ also being close to zero. Once the feature-positive discriminations are solved, the associative strength of $\mathrm{A}$ and $\mathrm{B}$ will be close to $1 \lambda$. Equation 5, therefore, predicts that $\sigma$ for $\mathrm{A}$ and $\mathrm{B}$ will be driven to its minimum value (.3), and Equation 2a predicts that $\alpha$ for $\mathrm{A}$ and $\mathrm{B}$ will be close to 1 . The product of $\alpha$ and $\sigma$ for A and B will therefore be approximately .3, which is higher than the total associability of $\mathrm{X}$ and $\mathrm{Y}$. The higher overall associability of $\mathrm{A}$ and $\mathrm{B}$ rather than of $\mathrm{X}$ and $\mathrm{Y}$ will then ensure that the discrimination between AY and BY will proceed more rapidly than the discrimination between AY and AX. A summary of these principles is shown in the bottom rows of Table 2. We should emphasize that the resolution of the apparent conflict between Experiments 1 and 3 described here can also be applied to Experiments 2 and 4 . We should also emphasize that this is by no means the only resolution. The hybrid model proposed by, for example, Le Pelley (2004, pp. 225-235, in press) provides a

\footnotetext{
${ }^{2}$ Equation 4 is based on the equation provided by Pearce and Hall (1980) to describe associability changes. As it is, it makes the unrealistic prediction that stimulus associability is equal to the prediction error from the immediately preceding trial. This equation was modified by Pearce, Kaye, and Hall (1982) so that changes in stimulus associability take place at a slower rate. We adopt the equation provided by Pearce and Hall simply for the sake of clarity. Le Pelley (2004, in press) showed simulations of a hybrid model that incorporates the equations proposed by Pearce et al. for determining stimulus associability.

${ }^{3}$ The terminal associative strength for partially reinforced CSs $\mathrm{X}$ and $\mathrm{Y}$ is influenced by the values of $\beta$ for reinforced $\left(\beta_{E}\right)$ and nonreinforced $\left(\beta_{I}\right)$ trials. If $\beta_{E}=\beta_{I}$, then $V_{X}$ and $V_{Y}$ will reach an asymptote of .5 $\lambda$. If $\beta_{E}>$ $\beta_{I}$, then $V_{X}$ and $V_{Y}$ will reach an asymptote greater than .5 . Finally, if $\beta_{E}<\beta_{I}$, then $V_{X}$ and $V_{Y}$ will reach an asymptote less than .5 $\lambda$. For the sake of simplicity, we assume in this analysis that $\beta_{\mathrm{E}}=\beta_{\mathrm{I}}$.
} 
similar analysis for these contrasting results. We have focused here on the analysis that can be derived from Pearce and Mackintosh (in press), only because it provides a simple integration of the proposals of Mackintosh (1975) and Pearce and Hall (1980).

The current experiments are consistent with the idea that two processes contribute to the overall associability of a CS. Experiments 1 and 2 are consistent with the Pearce-Hall (1980) model of learning; Experiments 3 and 4 are consistent with the Mackintosh (1975) model. The conditions of, in particular, Experiments 2 and 3 were extremely similar; we are therefore encouraged to attribute the different experimental outcomes to the impact of the differing pretraining rather than any other procedural differences between the experiments. To resolve the contradictory findings, we advocate a hybrid model approach to conditioning, in which the contribution of the Mackintosh and Pearce-Hall processes of attention are assumed to operate simultaneously on any conditioning trial.

\section{References}

Ayres, J. J. B., Moore, J. W., \& Vigorito, M. (1985). Hall and Pearce negative transfer: Assessments in conditioned suppression and nictitating membrane conditioning experiments. Animal Learning \& Behavior, 12, 428-438.

Bush, R. R., \& Mosteller, F. (1951). A mathematical model for simple learning. Psychological Review, 58, 313-323.

Dickinson, A. (1980). Contemporary animal learning theory. Cambridge, England: Cambridge University Press.

Dopson, J. C., Esber, G. R., \& Pearce, J. M. (2010). Differences in the associability of relevant and irrelevant stimuli. Journal of Experimental Psychology: Animal Behaviour Processes, 36, 258-267.

Hall, G. (2003). Learned changes in the sensitivity of stimulus representations: Associative and nonassociative mechanisms. Quarterly Journal of Experimental Psychology: Comparative and Physiological Psychology, 56(B), 43-55.

Hall, G., \& Pearce, J. M. (1979). Latent inhibition of a CS during CS-US pairings. Journal of Experimental Psychology: Animal Behavior Processes, 3, 31-42.

Hogarth, L., \& Duka, T. (2006). Human nicotine conditioning requires explicit contingency knowledge: Is addictive behaviour cognitively mediated? Psychopharmacology, 184, 553-566.

Honey, R. C., Good, M., \& Manser, K. L. (1998). Negative priming in associative learning: Evidence from a serial-habituation procedure. Journal of Experimental Psychology: Animal Behavior Processes, 24, 229-237.

Kamin, L. J. (1968). "Attention-like" processes in classical conditioning. In M. R. Jones (Ed.), Miami Symposium on the prediction of behavior: Aversive stimulation (pp. 9-31). Miami, FL: University of Miami Press.

Kaye, H., \& Pearce, J. M. (1984). The strength of the orienting response during Pavlovian conditioning. Journal of Experimental Psychology: Animal Behavior Processes, 10, 90-109.

Kruschke, J. K. (2001). Toward a unified model of attention in associative learning. Journal of Mathematical Psychology, 45, 812-863.

Le Pelley, M. E. (2004). The role of associative history in models of associative learning: A selective review and a hybrid model. Quarterly Journal of Experimental Psychology: Comparative and Physiological Psychology, 57(B), 193-243.

Le Pelley, M. E. (in press). The hybrid modelling approach to conditioning. In N. A. Schmajuk (Ed.), Computational models of conditioning. Cambridge, England: Cambridge University Press.

Le Pelley, M. E., \& Beesley, T. (2008, July). Predictive accuracy and stimulus associability: Testing the Pearce-Hall model in humans. Paper presented at the meeting of the Experimental Psychology Society, Liverpool, England.
Lovejoy, E. (1968). Attention in discrimination learning. San Francisco, CA: Holden-Day.

Mackintosh, N. J. (1975). A theory of attention: Variations in the associability of stimuli with reinforcement. Psychological Review, 82, 276-298.

Maddux, J.-M., Kerfoot, E. C., Chatterjee, S., \& Holland, P. C. (2007). Dissociation of attention in learning and action: Effects of lesions of the amygdala central nucleus, medial prefrontal cortex, and posterior parietal cortex. Behavioral Neuroscience, 121, 63-79.

Pavlov, I. P. (1927). Conditioned reflexes. London, England: Oxford University Press.

Pearce, J. M. (1987). A model for stimulus generalization in Pavlovian conditioning. Psychological Review, 94, 61-73.

Pearce, J. M. (2008). Animal learning and cognition. Hove, England: Psychology Press.

Pearce, J. M., Esber, G. R., George, D. N., \& Haselgrove, M. (2008). The nature of discrimination learning in pigeons. Learning and Behavior, 36, 188-199.

Pearce, J. M., George, D. N., \& Redhead, E. S. (1998). The role of attention in the solution of conditional discriminations. In N. A. Schmajuk \& P. C. Holland (Eds.), Occasion setting: Associative learning and cognition in animals (pp. 249-275). Washington, DC: American Psychological Association.

Pearce, J. M., \& Hall, G. (1980). A model for Pavlovian conditioning: Variations in the effectiveness of conditioned but not of unconditioned stimuli. Psychological Review, 87, 532-552.

Pearce, J. M., Kaye, H., \& Hall, G. (1982). Predictive accuracy and stimulus associability: Development of a model of associative learning. In M. Commons, R. Herrnstein, \& A. R. Wagner (Eds.), Quantitative analysis of behaviour (Vol. 3, pp. 241-256). Cambridge, MA: Ballinger.

Pearce, J. M., \& Mackintosh, N. J. (in press). Two theories of attention: A review and a possible integration. In M. E. Le Pelley \& C. J. Mitchell (Eds.), Learning and attention. Oxford, England: Oxford University Press.

Pearce, J. M., Wilson, P. N., \& Kaye, H. (1988). The influence of predictive accuracy on serial conditioning in the rat. Quarterly Journal of Experimental Psychology: Comparative and Physiological Psychology, 40(B), 181-198.

Rescorla, R. A. (1968). Probability of shock in the presence and absence of CS in fear conditioning. Journal of Comparative and Physiological Psychology, 66, 1-5.

Rescorla, R. A. (2000). Associative changes in excitors and inhibitors differ when they are conditioned in compound. Journal of Experimental Psychology: Animal Behavior Processes, 26, 428-438.

Rescorla, R. A. (2001). Unequal associative changes when excitors and neutral stimuli are conditioned in compound. Quarterly Journal of Experimental Psychology: Comparative and Physiological Psychology, 54(B), 53-68.

Rescorla, R. A. (2002). Effect of following an excitatory-inhibitory compound with an intermediate reinforcer. Journal of Experimental Psychology: Animal Behavior Processes, 28, 163-174.

Rescorla, R. A., \& Wagner, A. R. (1972). A theory of Pavlovian conditioning: Variations in the effectiveness of reinforcement and nonreinforcement. In A. H. Black \& W. F. Prokasy (Eds.), Classical conditioning II: Current research and theory (pp. 64-99). New York: Appleton-Century-Crofts.

Robinson, T. E., \& Berridge, K. C. (1993). The neural basis of drug craving: An incentive-sensitization theory of drug addiction. Brain Research Reviews, 18, 247-291.

Savastano, H. I., Yin, H., Barnet, R. C., \& Miller, R. R. (1998). Temporal coding in Pavlovian conditioning: Hall-Pearce negative transfer. Quarterly Journal of Experimental Psychology: Comparative and Physiological Psychology, 51(B), 139-153.

Schmajuk, N. A., Lam, Y. W., \& Gray, J. A. (1996). Latent inhibition: A neural network approach. Journal of Experimental Psychology: Animal Behavior Processes, 22, 321-349. 
Schmajuk, N. A., Lamoureux, J. A., \& Holland, P. C. (1998). Occasion setting: A neural network approach. Psychological Review, 105, 3-32.

Sutherland, N. S., \& Mackintosh, N. J. (1971). Mechanisms of animal discrimination learning. New York, NY: Academic Press.

Swan, J. A., \& Pearce, J. M. (1988). The orienting response as an index of stimulus associability in rats. Journal of Experimental Psychology: Animal Behavior Processes, 4, 292-301.

Swartzentruber, D., \& Bouton, M. E. (1986). Contextual control of negative transfer produced by prior CS-US pairings. Learning and Motivation, 17, 366-385.

Wagner, A. R., \& Rescorla, R. A. (1972). Inhibition in Pavlovian conditioning: Application of a theory. In R. A. Boakes \& M. S. Halliday (Eds.), Inhibition and learning (pp. 301-336). New York, NY: Academic Press.
Wilson, P. N., Boumphrey, P., \& Pearce, J. M. (1992). Restoration of the orienting response to a light by a change in its predictive accuracy. Quarterly Journal of Experimental Psychology: Comparative and Physiological Psychology, 44(B), 17-36.

Zeaman, D., \& House, B. J. (1963). The role of attention in retardate learning. In N. R. Ellis (Ed.), Handbook of mental deficiency: Psychological theory and research (pp. 159-223). New York, NY: McGrawHill.

Received July 10, 2009 Revision received November 19, 2009 Accepted November 24, 2009

\section{Call for Nominations}

The Publications and Communications (P\&C) Board of the American Psychological Association has opened nominations for the editorships of Journal of Experimental Psychology: Learning, Memory, and Cognition; Professional Psychology: Research and Practice; Psychology, Public Policy, and Law; and School Psychology Quarterly for the years 2013-2018. Randi C. Martin, $\mathrm{PhD}$, Michael C. Roberts, PhD, Ronald Roesch, PhD, and Randy W. Kamphaus, PhD, respectively, are the incumbent editors.

Candidates should be members of APA and should be available to start receiving manuscripts in early 2012 to prepare for issues published in 2013. Please note that the P\&C Board encourages participation by members of underrepresented groups in the publication process and would particularly welcome such nominees. Self-nominations are also encouraged.

Search chairs have been appointed as follows:

- Journal of Experimental Psychology: Learning, Memory, and Cognition, Leah Light, $\mathrm{PhD}$, and Valerie Reyna, $\mathrm{PhD}$

- Professional Psychology: Research and Practice, Bob Frank, PhD, and Lillian Comas-Diaz, $\mathrm{PhD}$

- Psychology and Aging, Leah Light, $\mathrm{PhD}$

- Psychology, Public Policy, and Law, Peter Ornstein, PhD, and Brad Hesse, PhD

- School Psychology Quarterly, Neal Schmitt, PhD, and Jennifer Crocker, PhD

Candidates should be nominated by accessing APA's EditorQuest site on the Web. Using your Web browser, go to http://editorquest.apa.org. On the Home menu on the left, find "Guests." Next, click on the link "Submit a Nomination," enter your nominee's information, and click "Submit."

Prepared statements of one page or less in support of a nominee can also be submitted by e-mail to Sarah Wiederkehr, P\&C Board Search Liaison, at swiederkehr@apa.org.

Deadline for accepting nominations is January 10, 2011, when reviews will begin. 